\title{
MULTILINEAR SINGULAR INTEGRALS
}

\author{
Christoph M. Thiele
}

\begin{abstract}
We survey the thoery of multilinear singular integral operators with modulation symmetry. The basic example for this theory is the bilinear Hilbert transform and its multilinear variants. We outline a proof of boundedness of Carleson's operator which shows the close connection of this operator to multilinear singular integrals. We discuss particular multilinear singular integrals which historically arose in the study of eigenfunctions of Schrödinger operators.
\end{abstract}

This survey article arose from a series of three expository lectures given at the 6th International Conference on Harmonic Analysis and Partial Differential Equations at El Escorial 2000. I would like to thank the organizers for organizing this stimulating and successful conference.

The article is divided into three chapters following the three lectures at El Escorial. The first section gives an introduction into the subject of multilinear singular integrals with modulation symmetries as it has evolved over the past five years. The second section presents a proof of boundedness of the Carleson operator, the essential ingredient in the proof of Carleson's theorem on almost everywhere convergence of Fourier series. We follow closely the work [29], with additional comments as presented during the lecture. While Carleson's operator is not a multilinear operator itself, it serves as a good model for the type of arguments used in the theory: thus the theory extends to other objects than multilinear operators.

The third section is again purely expository and gives an overview of how the discussed theory of multilinear singular integrals plays a role in eigenfunction expansions of one dimensional Schrödinger operators. In fact, some of the most prominent objects in the theory such as the bilinear Hilbert transform and Carleson's operator enter directly into these multilinear expansions.

2000 Mathematics Subject Classification. 42B20.

Key words. Multilinear operators, singular integrals, time-frequency analysis, Fourier series. 
The author thanks the organizers of the conference at El Escorial for their support. The author was partially supported by NSF grants DMS 9970469 and DMS 9985772 and by a Sloan Fellowship. The author thanks the referee for a list of corrections to the first manuscript.

\section{Theory of multilinear singular integrals}

\subsection{Littlewood-Paley theory.}

We start our discussion of multilinear singular integrals with an outline of an essential ingredient: Littlewood-Paley theory. While Littlewood-Paley theory appears as an essential tool for singular integrals in the linear and multilinear setting, it has its natural limitations in dealing with modulation invariant operators, which are our focus in the multilinear theory. In the modulation invariant setting one has to add further machinery to Littlewood-Paley theory, which will be one of the main points in these lectures.

We describe the most basic concepts of Littlewood-Paley theory using the example of the Hilbert transform. This central object of harmonic analysis is a linear operator acting on functions on the real line defined by

$$
H f(x):=\text { p.v. } \int_{\mathbb{R}} f(x-t) \frac{1}{t} d t .
$$

Alternatively one can write

$$
H:=-\mathcal{F}^{-1} M_{\text {sign }} \mathcal{F},
$$

where $\mathcal{F}$ is the Fourier transform defined by

$$
\mathcal{F} f(x):=\int_{\mathbb{R}} f(x) e^{-i \xi x} d x,
$$

and

$$
M_{\text {sign }} f(x):=-i \pi \operatorname{sign}(x) f(x) .
$$

The Hilbert transform is the prototype in one dimension of what is called a singular integral. For general reference see [37] and its predecessors. In this survey, we shall only be concerned with the one-dimensional theory, although eventually $\mathbb{R}^{n}$ will come in as $n$-fold tensor product of the onedimensional $\mathbb{R}^{1}$ when we discuss multilinear operators.

As written down, the Hilbert transform is defined on the space of Schwartz functions and has values in the space of tempered distributions. However, it satisfies the regularity estimate

$$
\|H f\|_{p} \leq C_{p}\|f\|_{p}
$$


for all $1<p<\infty$, where $C_{p}$ is a universal constant and

$$
\|f\|_{p}:=\left(\int_{\mathbb{R}}|f(x)|^{p} d x\right)^{\frac{1}{p}} .
$$

This result is due to Kolmogorov [22] and Riesz [36]. Hence the Hilbert transform extends to a bounded operator from $L^{p}$ to itself. This result is most easily seen for $p=2$ from the formula (2), since $\mathcal{F}$ is an isometry of the Hilbert space $L^{2}$, and multiplication by the function sign is also an isometry of $L^{2}$.

We shall look at the $L^{p}$ theory in the framework of Littlewood Paley theory. Consider the closely related multiplier operator $P_{+}$defined as in (1) where $M$ is defined by the characteristic function $1_{\mathbb{R}_{+}}$instead of the function $i \pi \operatorname{sign}$ in (2).

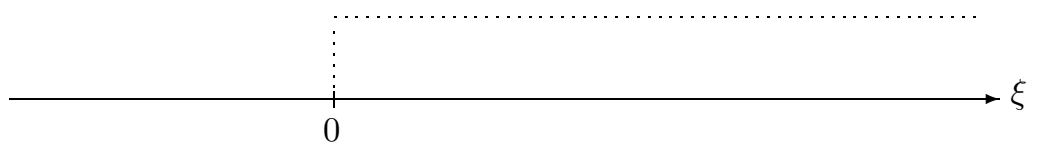

The main idea of Littlewood-Paley theory is to decompose the positive half line dyadically (powers of 2):

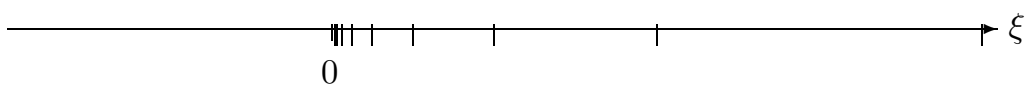

and then write the characteristic function of the half line as a sum of smooth functions adapted to these dyadic intervals:

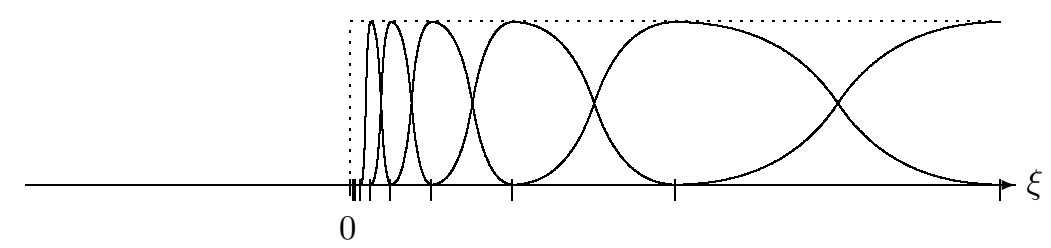

E.g., these summands can be chosen as dilates of each other:

$$
1_{\mathbb{R}_{+}}(\xi)=\sum_{k \in Z} \widehat{\phi}\left(2^{k} \xi\right) .
$$

The multiplier operator $P_{+}$can then be written as

$$
P_{+} f=\sum_{k \in \mathbb{Z}} \phi_{k} * f, \quad \phi_{k}(x)=2^{k} \phi\left(2^{k} x\right) .
$$


The basic inequality of Littlewood-Paley theory says that

$$
\left\|\left(\sum_{k \in \mathbb{Z}}\left|\phi_{k} * f\right|^{2}\right)^{\frac{1}{2}}\right\|_{p} \leq C_{p}\|f\|_{p}
$$

for all $1<p<\infty$. Using this, one can easily prove the KolmogorovRiesz theorem for $1<p<\infty$. With $p^{\prime}=p /(p-1)$ the dual exponent of $p$, we have to prove

$$
\left\langle P_{+} f, g\right\rangle \leq C_{p}\|f\|_{p}\|g\|_{p^{\prime}}
$$

for arbitrary $f$ and $g$. By Plancherel we have

$$
\left\langle P_{+} f, g\right\rangle=\left\langle 1_{R_{+}} \widehat{f}, 1_{R_{+}} \widehat{g}\right\rangle=\sum_{k, k^{\prime}}\left\langle\widehat{f} \widehat{\phi}_{k}, \widehat{g} \widehat{\phi}_{k^{\prime}}\right\rangle .
$$

Considering the supports of $\phi_{k}$ and $\phi_{k^{\prime}}$, we may assume that $k$ and $k^{\prime}$ differ by at most 1 . By splitting into cases we may assume that the difference is constant, for simplicity we discuss only $k=k^{\prime}$. Then we have by Plancherel, Cauchy Schwarz, and Hölder:

$$
\begin{aligned}
& =\left|\sum_{k}\left\langle f * \phi_{k}, g * \phi_{k}\right\rangle\right| \\
& \leq \int\left(\sum_{k \in \mathbb{Z}}\left|\phi_{k} * f\right|^{2}\right)^{\frac{1}{2}}\left(\sum_{k \in \mathbb{Z}}\left|\phi_{k} * g\right|^{2}\right)^{\frac{1}{2}} d x \\
& \leq\left\|\left(\sum_{k \in \mathbb{Z}}\left|\phi_{k} * f\right|^{2}\right)^{\frac{1}{2}}\right\|\left\|\left(\sum_{k \in \mathbb{Z}}\left|\phi_{k} * g\right|^{2}\right)^{\frac{1}{2}}\right\|_{p} .
\end{aligned}
$$

By the basic Littlewood-Paley inequality this is bounded by $\|f\|_{p}\|g\|_{p^{\prime}}$.

Generally, the idea of Littlewood-Paley theory is to use dyadic frequency decompositions to simplify operators and obtain estimates such as the above Littlewood-Paley inequality. We shall see momentarily that this idea remains important but shall have its limitations in the modulation invariant setting, because it singles out the 0 frequency as special.

\subsection{Modulation invariant operators.}

Now we shall consider operations with modulation symmetries. Modulation is defined by multiplication with a complex exponential:

$$
M_{\xi} f(x):=f(x) e^{2 \pi i \xi x} .
$$

This amounts to a translation of the Fourier transform of $f$. 
The bilinear Hilbert transform $H_{\alpha}$ with parameter $\alpha \in \mathbb{R}$ is a bilinear operator mapping two functions on the real line to one function on the real line. It is defined by

$$
H_{\alpha}(f, g)(x):=\text { p.v. } \int_{\mathbb{R}} f(x-t) g(x-\alpha t) \frac{1}{t} d t .
$$

Again, this expression is well defined if $f$ and $g$ are Schwartz functions. An easy calculation shows the following modulation symmetry:

$$
B\left(M_{\alpha \xi} f, M_{-\xi} g\right)=M_{(\alpha-1) \xi} B(f, g) .
$$

Another example for a modulation invariant operator is Carleson's operator:

$$
C(f)(x)=\sup _{\xi}\left|\int_{-\infty}^{\xi} \widehat{f}(\xi) e^{2 \pi i \xi x} d \xi\right| .
$$

It is a maximal variant of the Hilbert transform and plays a role in almost everywhere convergence of Fourier series. It satisfies

$$
C\left(M_{\xi} f\right)=C(f) \text {. }
$$

We now make the important observation that a Littlewood-Paley decomposition

$$
f=\sum_{k \in Z} f * \phi_{k}
$$

does not break up these modulation invariant operators into simpler pieces. As an example consider Carleson's operator. Assume we could estimate each single piece of the Littlewood-Paley decomposition. Assume w.l.o.g. that $\widehat{f}$ is compactly supported. Then for some large $\xi$ and $k$

$$
C(f)=C\left(M_{\xi} f\right)=C\left(\phi_{k} * M_{\xi} f\right) .
$$

(Here we assume $\widehat{\phi_{k}}$ to be constant equal to 1 on some small interval.) In other words, we can move the function $f$ in frequency until it lies entirely inside a single block of the Littlewood Paley decomposition. Clearly this makes the Littlewood-Paley decomposition useless at this point. The individual pieces of Carleson's operator with respect to a Littlewood-Paley decomposition are as hard to estimate as the whole operator. Another way to look at this has been mentioned before: the Littlewood-Paley decomposition does single out the zero frequency as special, which cannot do good in the modulation invariant setting. For the same reason, spaces like $H^{1}$ and BMO, which single out the zero frequency as special, play a less prominent role in the modulation invariant setting. 
While an outright Littlewood-Paley decomposition does not do anything to modulation invariant operators, these operators still have an $L^{p}$ theory (which in fact uses portions of Littlewood-Paley theory at the right places). We shall postpone any further details to the next chapter and continue here with an outline of the theory.

The first result of interest for our discussion is the Carleson-Hunt theorem

Theorem 1.1. The Carleson operator is bounded in $L^{p}$ for $1<p<\infty$,

$$
\|C f\|_{p} \leq C_{p}\|f\|_{p} .
$$

The weak type 2 estimate $[6]$ by L. Carleson was a major breakthrough and pioneering work on modulation invariant operators. Later, R. A. Hunt proved boundedness of $C$ in all $L^{p}[\mathbf{1 8}]$. Further proofs of Carleson's theorem appeared in $[\mathbf{1 2}]$ and $[\mathbf{2 9}]$. We will present a proof of Carleson's theorem in the second chapter. This will also serve as our main description of the type of techniques used to estimate modulation invariant singular integral operators.

Estimates for the bilinear Hilbert transform are more recent. In the series of papers $[\mathbf{2 5}],[\mathbf{2 6}],[\mathbf{2 7}]$, and [28], it has been proved that the bilinear Hilbert transform satisfies the following regularity estimates:

Theorem 1.2. If $\alpha \notin\{0,1\}$, then

$$
\left\|H_{\alpha}\left(f_{1}, f_{2}\right)\right\|_{p} \leq C_{p_{1}, p_{2}}\left\|f_{1}\right\|_{p_{1}}\left\|f_{2}\right\|_{p_{2}},
$$

provided that

$$
\begin{gathered}
\frac{1}{p}=\frac{1}{p_{1}}+\frac{1}{p_{2}}, \\
\frac{2}{3}<p<\infty, \\
1<p_{1}, p_{2} \leq \infty .
\end{gathered}
$$

A precedent of this work is $[\mathbf{2 3}]$. In contrast to the situation for the Hilbert transform $H$, none of the estimates in this theorem is particularly easy to prove. For $\alpha \in\{0,1\}$ we observe

$$
\begin{aligned}
& H_{0}(f, g)=H(f) \cdot g, \\
& H_{1}(f, g)=H(f \cdot g) .
\end{aligned}
$$


In these cases one easily obtains regularity results (but in different regions than described in the theorem) by an application of the theorem of Kolmogorov and Riesz and by Hölder's inequality.

The study of the bilinear Hilbert transform can be simplified by taking into account a symmetry which is not immediately apparent from the expression (5). To exploit this symmetry, consider the trilinear form

$$
\Lambda_{(\alpha)}\left(f_{1}, f_{2}, f_{3}\right):=\int_{\mathbb{R}} H_{\alpha}\left(f_{1}, f_{2}\right)(x) f_{3}(x) d x
$$

For each $\alpha$, this trilinear form is a member of the following family of forms

$$
\Lambda_{\beta}\left(f_{1}, f_{2}, f_{3}\right):=\int \text { p.v. } \int\left(\prod_{\jmath=1}^{3} f_{\jmath}\left(x-\beta_{\jmath} t\right)\right) \frac{1}{t} d t d x
$$

where $\beta=\left(\beta_{1}, \beta_{2}, \beta_{3}\right)$ is a vector in $\mathbb{R}^{3}$ which is not collinear with $\gamma:=(1,1,1)$. By a change of variables it is no restriction to assume that $\beta$ is a unit vector perpendicular to the vector $\gamma$. Hence the family $\Lambda_{\beta}$ is a one parameter family which consists of all forms which are dual in the sense of (8) to $\pm H_{\alpha}$ with $\alpha \in \mathbb{R}$ and the forms which are dual to

$$
\pm H_{\infty}\left(f_{1}, f_{2}\right):= \pm f_{1} \cdot H\left(f_{2}\right)
$$

The group of permutations of $\{1,2,3\}$ is acting in an obvious way on the family $\Lambda_{\beta}$, which is the symmetry mentioned above. We call $\beta$ nondegenerate if $\beta_{\imath} \neq \beta_{\jmath}$ for $\imath \neq \jmath$. If $\beta$ is non-degenerate, then $\Lambda_{\beta}$ is dual to $\pm H_{\alpha}$ for some $\alpha \notin\{0,1, \infty\}$.

Using the Fourier transform, we can rewrite (8) as a constant multiple of

$$
\int \delta\left(\xi_{1}+\xi_{2}+\xi_{3}\right) \widehat{f}_{1}\left(\xi_{1}\right) \widehat{f}_{2}\left(\xi_{2}\right) \widehat{f}_{3}\left(\xi_{3}\right) \operatorname{sgn}\left(\beta_{1} \xi_{1}+\beta_{2} \xi_{2}+\beta_{3} \xi_{3}\right) d \xi_{1} d \xi_{2} d \xi_{3}
$$

where $\delta$ is the Dirac $\delta$ function and thus the integration is over the plane $\xi_{1}+\xi_{2}+\xi_{3}=0$. 


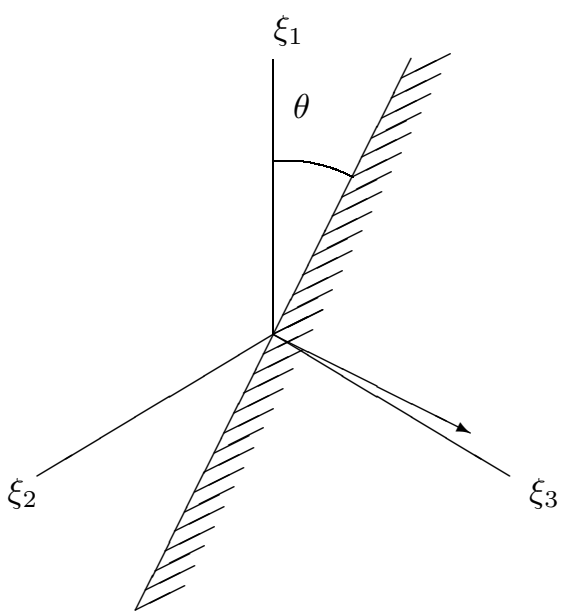

The estimates of Theorem 1.2 with $p \geq 1$ are equivalent (in the case $p=1$ this is only almost true) to certain estimates for the form $\Lambda_{\beta}$ by using the duality of $L^{p}$ and $L^{p^{\prime}}$ where $p^{\prime}$ denotes the conjugate exponent of $p$,

$$
\frac{1}{p^{\prime}}+\frac{1}{p}=1
$$

The estimates of Theorem 1.2 with $p<1$ can not be stated as estimates for $\Lambda_{\beta}$. Hence we define formally the following notion of type: For each permutation $\sigma$ of $\{1,2,3\}$ define the bilinear operator $H_{\beta}^{\sigma}$ formally by

$$
H_{\beta}^{\sigma}: S(\mathbb{R}) \times S(\mathbb{R}) \rightarrow S^{\prime}(\mathbb{R})
$$

by:

$$
H_{\beta}^{\sigma}\left(f_{\sigma(1)}, f_{\sigma(2)}\right)\left(f_{\sigma(3)}\right):=\Lambda_{\beta}\left(f_{1}, f_{2}, f_{3}\right) .
$$

We say that $\Lambda_{\beta}$ is of type $\left(p_{1}, p_{2}, p_{3}\right)$ if there is a permutation $\sigma$ such that

$$
0<p_{\sigma(1)}, p_{\sigma(2)}, p_{\sigma(3)}{ }^{\prime} \leq \infty
$$

and the operator $H_{\beta}^{\sigma}$ is bounded from $L^{p_{\sigma(1)}} \times L^{p_{\sigma(2)}}$ to $L^{p_{\sigma(3)}{ }^{\prime}}$. This notion of type makes sense when (at most) one of the $p_{i}$ is negative: then the dual $p_{i}^{\prime}$ is between 0 and 1 and the estimate for the corresponding dual bilinear operator makes sense. If all $p_{i}$ are betwen 1 and $\infty$, the actual choice of the dual bilinear operator plays no role.

Theorem 1.2 states that $\Lambda_{\beta}$ is of the type $\left(p_{1}, p_{2}, p_{3}\right)$ whenever $\left(1 / p_{1}, 1 / p_{2}, 1 / p_{3}\right)$ is in the region described by the following diagram: 
$(0,0,1)$

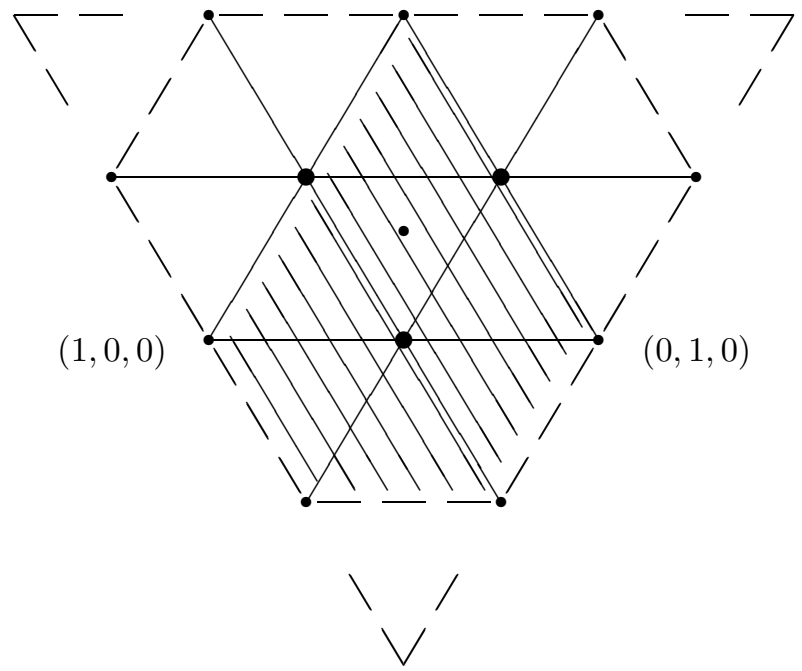

Observe that $p_{3}{ }^{\prime}<1$ means $p_{3}<0$. The region where $1<p_{i}<\infty$ for all $i$ is the convex hull of the indicated points.

By self duality of the family $\Lambda_{\beta}$, more estimates (types) are true than stated in Theorem 1.2. We obtain the convex hull of the indicated regions:

$$
(1,0,0)
$$
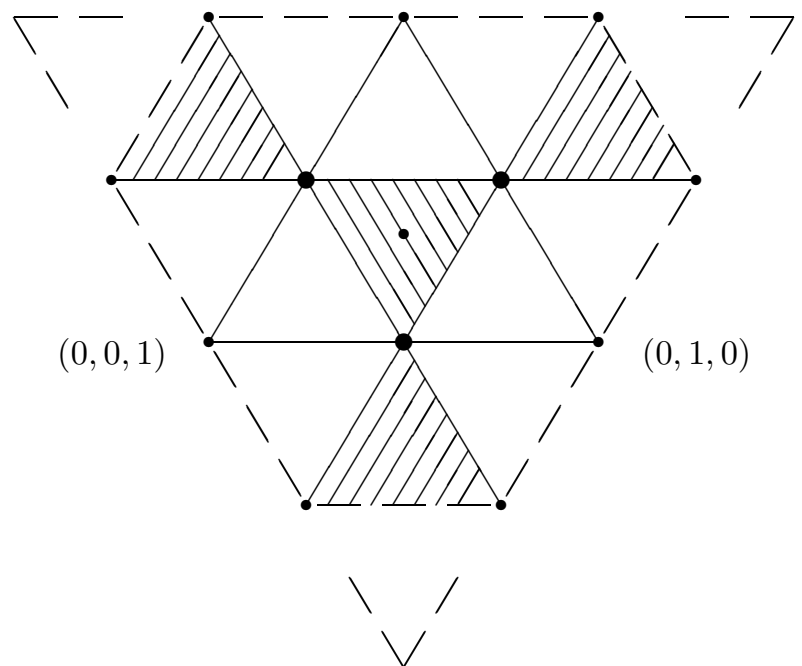
In fact, it suffices to prove estimates only in the shaded regions, the estimates in the convex hull then follow by interpolation methods $[\mathbf{5}],[\mathbf{1 9}],[\mathbf{3 3}]$. The articles $[\mathbf{2 5}]$ and $[\mathbf{2 6}]$ are concerned with the inner triangle, while $[\mathbf{2 7}]$ and $[\mathbf{2 8}]$ are concerned with the outer triangles.

Thus a corollary of Theorem 1.2 is that for non-degenerate $\beta$ the form $\Lambda_{\beta}$ is of type $\left(p_{1}, p_{2}, p_{3}\right)$ if

$$
\frac{1}{p_{1}}+\frac{1}{p_{2}}+\frac{1}{p_{3}}=1, \quad-\frac{1}{2}<\frac{1}{p_{1}}, \frac{1}{p_{2}}, \frac{1}{p_{3}}<1
$$

It is not known, whether the lower bound $\frac{2}{3}$ on $p$ in Theorem 1.2 can be relaxed to $\frac{1}{2}$. If this was true, we had estimates in the interior of the whole triangle shown by the above diagram.

In [13], Gilbert and Nahmod have extended the result of Theorem 1.2 to bilinear operators given by more general multipliers. We shall define these operators in terms of their dual trilinear forms. These forms are given by

$$
\begin{aligned}
& \Lambda_{m}\left(f_{1}, f_{2}, f_{3}\right) \\
& \quad=\int \delta\left(\xi_{1}+\xi_{2}+\xi_{3}\right) \widehat{f}_{1}\left(\xi_{1}\right) \widehat{f}_{2}\left(\xi_{2}\right) \widehat{f}_{3}\left(\xi_{3}\right) m\left(\xi_{1}, \xi_{2}, \xi_{3}\right) d \xi_{1} d \xi_{2} d \xi_{3},
\end{aligned}
$$

where $m$ is a function (multiplier) satisfying

$$
\left|\partial^{\alpha} m\left(\xi_{1}, \xi_{2}, \xi_{3}\right)\right| \leq C_{\alpha} \operatorname{dist}\left(\xi, \Gamma^{\prime}\right)^{-|\alpha|}
$$

and $\Gamma^{\prime}$ is some (non-degenerate as before) line in the plane $\xi_{1}+\xi_{2}+\xi_{3}=0$.

This condition on $m$ implies that $m$ is essentially constant on the Whitney-regions 


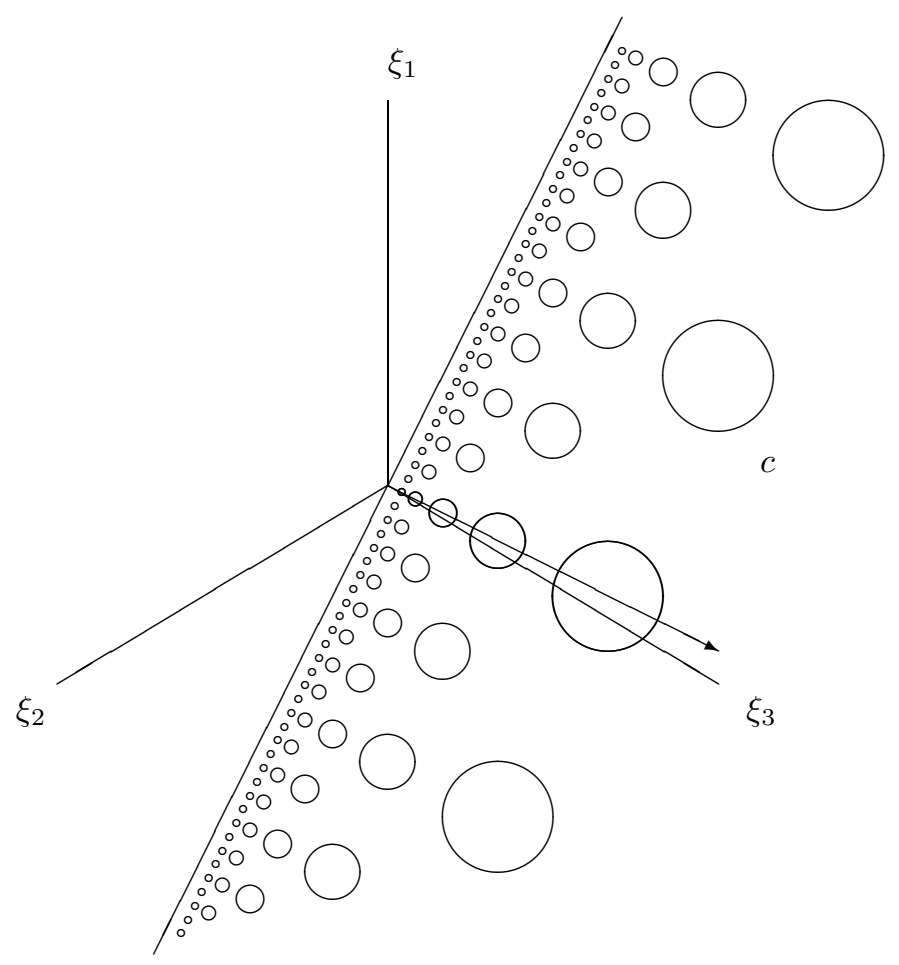

In fact, the proof of boundedness of these operators starts with a decomposition of the form $\Lambda$ into pieces parameterized by the Whitney regions:

$$
\Lambda_{m}\left(f_{1}, f_{2}, f_{3}\right) "=" \int \sum_{c}\left(f_{1} * \phi_{1, c}\right)\left(f_{2} * \phi_{2, c}\right)\left(f_{3}, \phi_{3, c}\right) d x .
$$

We have written this identity in quotation marks, since it is missing some error terms which are inessential for our discussion: in order to write the multiplier in the indicated form, we would have to write the characteristic function of the half plane as sum of smooth functions which are tensor products of functions in the variables $\xi_{1}, \xi_{2}$, and $\xi_{3}$, which requires a series of terms for each Whitney region $c$.

The similarity with the Littlewood-Paley decomposition of the previous section is now apparent. However, a simple Cauchy Schwartz argument does not apply here, for once we have three instead of two terms, 
and also because the functions $\phi_{i, c}$ not only capture dyadic frequency bands, but frequency bands of all possible length and location in frequency space. (The frequency interval of $\phi_{i, c}$ is given by projection of the Whitney region $c$ onto the $\xi_{i}$-axis; see the previous figure.) We will discuss how to deal with a similar expression for Carleson's operator in the next chapter.

\subsection{Uniform estimates.}

One of the degenerate cases $(\alpha=1)$ of the bilinear Hilbert transform is given by

$$
H_{1}\left(f_{1}, f_{2}\right)=H\left(f_{1} \cdot f_{2}\right)
$$

or its dual operators

$$
f_{2} \cdot H\left(f_{3}\right), \quad f_{1} \cdot H\left(f_{3}\right) .
$$

Besides the usual homogeneity, the only constraint for these operators to be bounded is $1<p_{3}<\infty$, i.e., one has estimates (types) in the horizontal open strip indicated in the following diagram:

$(0,0,1)$
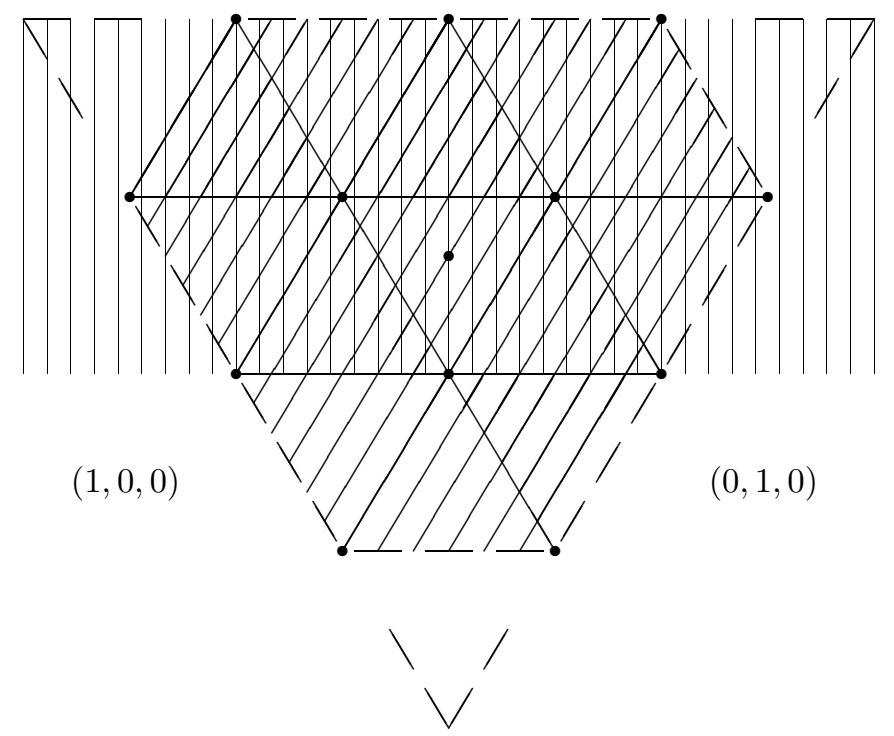
Thus one expects the constants in the $L^{p}$ estimates to be uniform as $\alpha$ approaches 1 in the intersection of the shaded hexagon and the shaded horizontal strip.

Uniformity of the constants does not come out of the original proofs of Theorem 1.2. Uniform estimates have been proved in [40], [16], and [30]. The following diagram indicates the known uniform estimates

$(0,0,1)$

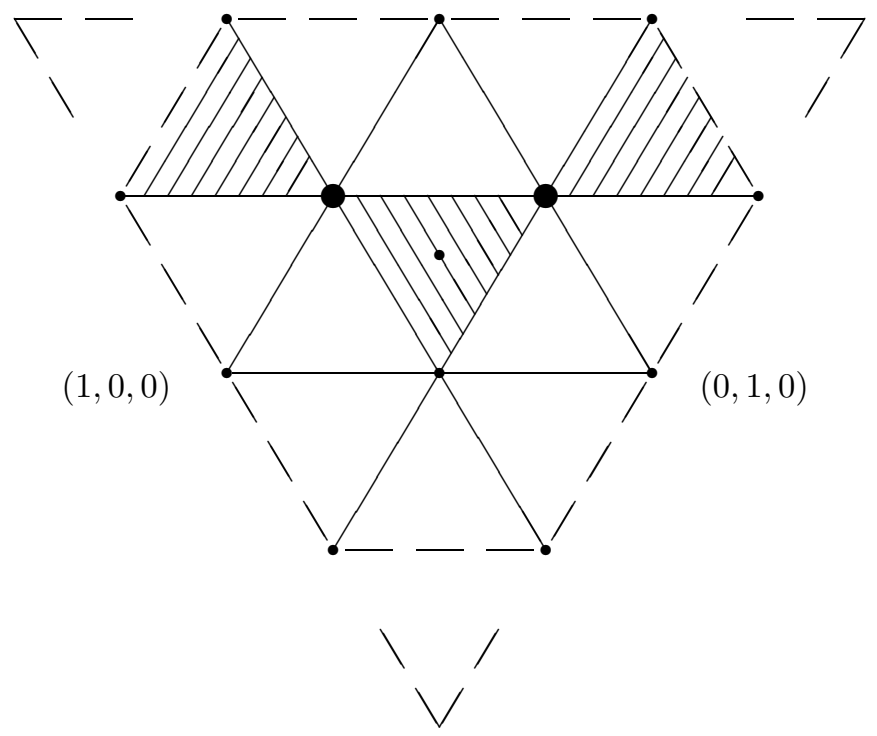

In [40], the weak type estimate $L^{2} \times L^{2} \rightarrow L^{1, \infty}$ is proved, i.e.,

$$
\left|\left\{x:\left|H_{\alpha}\left(f_{1}, f_{2}\right)\right|>\lambda\right\}\right| \leq C\left\|f_{1}\right\|_{2}\left\|f_{2}\right\|_{2}
$$

with a constant $C$ uniform in $\alpha$ near $\alpha=1$. With an unfortunately erroneous argument, it is claimed in [40] that one can bootstrap to the strong type estimate $L^{2} \times L^{2} \rightarrow L^{1}$, which together with a dual estimate would give the types indicated by the two large filled circles in the above diagram.

Grafakos and $\mathrm{Li}$ in [16] refine the arguments of [40] to give uniform estimates in the interior triangle. $\mathrm{Li}$ in $[\mathbf{3 0}]$ gives uniform estimates in the two indicated exterior triangles. The distinction of interior and exterior triangles is in analogy to [25] and [27]. By interpolation, one obtains 
the convex hull of the shaded regions, in particular those estimates that have been erroneously claimed in [40].

Observe that this convex hull still misses some part near $(1,0,0)$ and $(0,1,0)$, where according to a the above discussion one might expect uniform estimates. In these regions the question of uniformity is still open.

In order to prove uniform bounds, one has to formulate the multiplier condition so as to give essentially constant multipliers on adapted regions

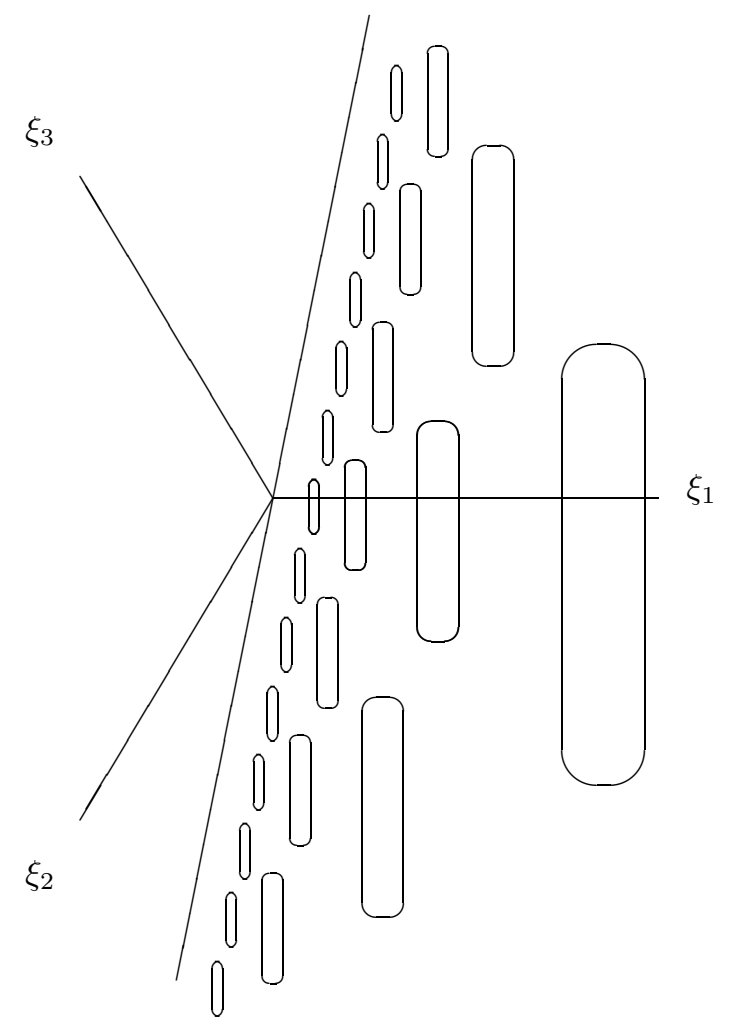

The excentricity of the ellipses is chosen so that the projections of the ellipses at a single scale onto any of the three axes $\xi_{1}, \xi_{2}, \xi_{3}$ form essentially a disjoint partition of the axis. In the degenerate case, which in this figure would mean a vertical dividing line, the ellipses become infinitely long. If one used Whitney circles as before, infinitely many circles would be projected to the same interval on the $\xi_{1}$-axis, which would 
make the arguments following the decomposition invalid. Dealing with ellipses rather than circles requires strong refinements of the arguments in $[\mathbf{2 5}]$ and $[\mathbf{2 7}]$.

Historically, the interest in the bilinear Hilbert transform arose from the study of the Cauchy integral and the Hilbert transform on Lipschitz curves and - as a first step in the study of these - the first commutator of Calderón. Let

$$
\gamma(x)=x+i A(x)
$$

be a curve in $\mathbb{C}$, where $A^{\prime}=a \in L^{\infty}(\mathbb{R})$. Then the Hilbert transform corresponding to the curve $\gamma$ is given by

$$
H_{\gamma} f(x):=\text { p.v. } \int \frac{f(y)(1+i a(y)) d y}{x-y+i(A(x)-A(y))} .
$$

It is a celebrated result by Calderón [4] (for small norm of $a$ ) and Coifman, McIntosh, Meyer [9] (for arbitrary norm of $a$ ), that this operator is bounded from $L^{2}(\mathbb{R})$ to itself.

One approach to this result is to expand the nonlinear dependence on $A$ into a Taylor series, i.e., to write the series expansion

$$
\frac{1}{x-y+i(A(x)-A(y))}=\frac{1}{x-y} \sum_{k=0}^{\infty}(-i)^{k}\left(\frac{A(x)-A(y)}{x-y}\right)^{k} .
$$

Ignoring the factor $1+i a(y)$ in the definition of $H_{\gamma}$, which is irrelevant for the question of boundedness in $L^{2}$, we are led to study the operators (commutators)

$$
\mathcal{C}_{k} f(x)=\text { p.v. } \int \frac{(A(x)-A(y))^{k}}{(x-y)^{k+1}} f(y) d y .
$$

Naturally, $\mathcal{C}_{0}$ is the Hilbert transform. For $\mathcal{C}_{1}$, Calderón's first commutator, we write

$$
\begin{aligned}
& \text { p.v. } \iint_{0}^{1} a(x+\alpha(y-x)) \frac{1}{x-y} f(y) d \alpha d y \\
= & \text { p.v. } \iint_{0}^{1} a(x-\alpha y) f(x-y) \frac{1}{y} d \alpha d y \\
= & \int_{0}^{1} H_{\alpha}(f, a)(x) d \alpha .
\end{aligned}
$$

Coming from the original question concerning the Hilbert transform on $\gamma$, one seeks for fixed $a \in L^{\infty}$ a bound on $\mathcal{C}_{1}$ as an operator from $L^{2}$ to itself. One of the attempts of Calderón was to prove appropriate bounds 
on the bilinear Hilbert transform, but he did not succeed this way and eventually proved boundedness of $\mathcal{C}_{1}$ by different means $[\mathbf{4}]$.

Using boundedness of the bilinear Hilbert transform one tries

$$
\left\|\int_{0}^{1} H_{\alpha}(f, a)(.) d \alpha\right\|_{2} \leq \int_{0}^{1}\left\|H_{\alpha}(f, a)\right\|_{2} d \alpha \leq \int_{0}^{1} C_{\alpha}\|f\|_{2}\|a\|_{\infty} d \alpha .
$$

The result of Theorem 1.2 does not give boundedness of $\mathcal{C}_{1}$, since it does not give good control over the constants $C_{\alpha}$ as $\alpha$ approaches 0 or 1 , indeed the proofs in [25] and [27] yield only polynomial growth of $C_{\alpha}$ in $\alpha, \alpha^{-1}$, and $(1-\alpha)^{-1}$.

However, the uniform results do show that in the above application $C_{\alpha}$ remains bounded for $\alpha$ near 0 and 1 , what is needed are the uniform estimates at the two large filled circles in the above diagram of uniform estimates. Thus one can conclude boundedness of the first commutator along these lines. Indeed, while the results in $[40]$ do not give strong type estimates, they can be bootstrapped to give strong type estimates with logarithmic blowup of $C_{\alpha}$ near $\alpha=0,1$, which still is enough to give integrability of $C_{\alpha}$ near 0 and 1 .

Closely related to the topic of uniform estimates for the bilinear Hilbert transform is that of bilinear multiplier estimates for multipliers which are singular along a curve rather than a line. Results in this direction are by C. Muscalu [32] and Grafakos and Li [17]. Consider as an example the trilinear form where $m\left(\xi_{1}, \xi_{2}, \xi_{3}\right)$ is the characteristic function of a disc. Suppose we have (as proved in [17]) $L^{p}$ bounds for this multiplier. By dilation and modulation symmetries we have the same bound for a multiplier which is the characteristic function of a different disc. Letting the boundary of the disc pass through the origin at a certain angle and taking a limit as the radius tends to infinity, we obtain the characteristic function of a half plane, thus reproving boundedness of the bilinear Hilbert transform at this angle. Since the angle was arbitrary, we obtain uniform estimates for all angles. Thus boundedness of the disc multiplier is stronger than uniform boundedness of the bilinear Hilbert transform. Grafakos and Li prove in [17] estimates for the disc multiplier that correspond to the region of exponents which was called the "inner triangle" above.

\subsection{Multilinear operators.}

In this section we discuss multilinear generalizations of the theory outlined above. We shall focus on a certain direction of generalization here; another direction will be discussed in the chapter on eigenfunction expansions of Schrödinger operators. 
For better symmetry we shall focus on the theory of multilinear forms. Consider general multilinear forms

$$
\Lambda: S(\mathbb{R}) \times \cdots \times S(\mathbb{R}) \times S(\mathbb{R}) \rightarrow \mathbb{C} .
$$

These correspond to multilinear operators $T$

$$
T: S(\mathbb{R}) \times \cdots \times S(\mathbb{R}) \rightarrow S^{\prime}(\mathbb{R})
$$

via duality:

$$
\Lambda\left(f_{1}, \ldots, f_{n}\right)=\int T\left(f_{1}, \ldots, f_{n-1}\right)(x) f_{n}(x) d x .
$$

Observe that the symmetric group on $n$ elements acts on the space of $n$-linear forms.

By the Schwartz kernel theorem, there is a distribution $m \in S^{\prime}\left(\mathbb{R}^{n}\right)$ so that

$$
\Lambda\left(f_{1}, \ldots, f_{n}\right)=\int m(\xi) \prod_{i=1}^{n} \widehat{f}_{i}\left(\xi_{i}\right) d \xi_{i} .
$$

We shall make the assumption that our multilinear forms are invariant under simultaneous translation of all $f_{i}$. This is a vary natural condition: it can be compared to the situation of differential operators with non-constant coefficients, which still have translation symmetries if one translates simultaneously the coefficients and the functions these operators act on.

Define the translation $T_{y}$ by

$$
T_{y} f(x)=f(x-y),
$$

then invariance of $\Lambda$ means

$$
\Lambda\left(T_{y} f_{1}, \ldots, T_{y} f_{n}\right)=\Lambda\left(f_{1}, \ldots, f_{n}\right) .
$$

For the distribution $m$ this implies

$$
m(\xi)=m(\xi) e^{2 \pi i y\left(\xi_{1}+\cdots+\xi_{n}\right)} .
$$

Hence $m$ is a distribution on the hyperplane

$$
\begin{gathered}
\Gamma:=\left\{\xi: \xi_{1}+\cdots+\xi_{n}=0\right\} \\
\Lambda\left(f_{1}, \ldots, f_{n}\right)=\int \delta\left(\xi_{1}+\cdots+\xi_{n}\right) m(\xi) \prod_{i=1}^{n} \widehat{f}_{i}\left(\xi_{i}\right) d \xi_{i} .
\end{gathered}
$$


The dual operator can then formally be written (identifying $m$ as a function in the variables $\xi_{1}, \ldots, \xi_{n-1}$ only)

(11) $T\left(f_{1}, \ldots, f_{n-1}\right)(x)=\int m\left(\xi_{1}, \ldots, \xi_{n-1}\right) e^{2 \pi i x\left(\xi_{1}+\cdots+\xi_{n}\right)} \prod_{i=1}^{n-1} \widehat{f}_{i}\left(\xi_{i}\right) d \xi_{i}$.

In the case of bilinear forms and linear operators this $m$ is the usual multiplier.

In order to study modulation symmetries, we choose a subspace

$$
\Gamma^{\prime} \subset \Gamma
$$

and demand $m$ to be invariant under all translations

$$
m=T_{\eta} m
$$

with $\eta \in \Gamma^{\prime}$. Since $m$ is a function in frequency space, this means a modulation symmetry for $\Lambda$ : it is invariant under certain simultaneous modulations of the arguments $f_{1}, \ldots, f_{2}$.

More generally, one may demand symbol estimates

$$
\left|\partial^{\alpha} m(\xi)\right| \leq C_{\alpha} \operatorname{dist}\left(\xi, \Gamma^{\prime}\right)^{-|\alpha|}
$$

for all partial derivatives $\partial^{\alpha}$ tangential to $\Gamma$. While an individual $m$ satisfying these estimates may not be translation invariant, certainly the whole class of such functions $m$ is translation invariant along $\Gamma^{\prime}$.

As is shown in [33], under a non-degeneracy condition on $\Gamma^{\prime}$ and for $\operatorname{dim}\left(\Gamma^{\prime}\right)<\frac{n}{2}$, one has in general $L^{p}$ estimates for such multilinear forms:

Theorem 1.3. Let $k, n$ be integers with

$$
0 \leq k<n / 2 \text {. }
$$

Let $\Gamma$ be the hyperplane in $\mathbb{R}^{n}$ as before and let $\Gamma^{\prime}$ be a $k$-dimensional subspace of $\Gamma$ such that the orthogonal projection of $\Gamma^{\prime}$ onto any $k d i$ mensional space spanned by $k$ coordinate axes is nondegenerate.

Let $m$ be a function on $\Gamma$ such that

$$
\left|\partial^{\alpha} m(\xi)\right| \leq \operatorname{dist}\left(\xi, \Gamma^{\prime}\right)^{-|\alpha|}
$$

for all partial derivatives tangential to $\Gamma$ up to some large finite order.

Then the corresponding $\Lambda$ satisfies

$$
\left|\Lambda\left(f_{1}, \ldots, f_{n}\right)\right| \leq C \prod_{i=1}^{n}\left\|f_{i}\right\|_{p_{i}}
$$

as long as

$$
\sum_{i=1}^{n} \frac{1}{p_{i}}=1, \quad 1<p_{1}, \ldots, p_{n}<\infty
$$


Even more is true: with the analogue notion of type as before, the form $\Lambda$ is of type $\left(p_{1}, \ldots, p_{n}\right)$, if $\sum_{i} 1 / p_{i}=1$, and $1<p_{i} \leq \infty$ for all $i$ with one possible exception, which may satisfy $-\infty<p_{i}<1 /(2-n)$, and we have

$$
\frac{1}{p_{i_{1}}}+\cdots+\frac{1}{p_{i_{r}}}<\frac{n-2 k+r}{2}
$$

for all $1 \leq i_{1}<\cdots<i_{r} \leq n$ and $1 \leq r \leq n$. In case $p=\infty$, the claim only stands for the closure of compactly supported functions in $L^{\infty}$ rather than $L^{\infty}$ itself.

The case $k=0$ of this theorem is well known. In fact it is the case of no modulation symmetries and can be treated in a direct manner with Littlewood-Paley theory. This theory has been pioneered by Coifman and Meyer, a nice exposition of this theory of multilinear operators can be found in $[\mathbf{3 1}]$.

The case $n=3$ of trilinear forms (bilinear operators) was proven by Gilbert and Nahmod [13], it includes the case of the bilinear Hilbert transform.

Observe that the four-linear form corresponding to what is called the trilinear Hilbert transform,

$$
H_{\alpha, \beta}(f, g, h)(x):=\text { p.v. } \int_{\mathbb{R}} f(x-y) g(x-\alpha t) h(x-\beta t) \frac{1}{t} d t,
$$

is given by a multiplier which is constant on two half spaces of the three dimensional space $\xi_{1}+\xi_{2}+\xi_{3}+\xi_{4}=0$, and thus does not fall into the scope of the above theorem. Namely, its parameters $k=2$ and $n=4$ violate the condition $2 k<n$. In fact, it is not known, whether this trilinear operator satisfies any $L^{p}$ estimates except in the degenerate cases $\alpha=\beta$ or $\{\alpha, \beta\} \cap\{0,1, \infty\} \neq \emptyset$.

If the multiplier $m$ in the above theorem is invariant under translations in direction of $\Gamma^{\prime}$, then we can formally take Fourier transforms and write the $n$-1-linear operator $T$ as

$$
T\left(f_{1}, \ldots, f_{n-1}\right)(x)=\int_{\Gamma^{\prime \prime} \cap \Gamma} f_{1}\left(x+\gamma_{1}\right) \ldots f_{n-1}\left(x+\gamma_{n-1}\right) K(\gamma) d \gamma
$$

where $\Gamma^{\prime \prime}$ is the orthogonal complement of $\Gamma^{\prime}$ in $\mathbb{R}^{n}, d \gamma^{\prime}$ is Lebesgue measure on $\Gamma^{\prime \prime} \cap \Gamma, \gamma_{i}$ is the $i$-th coordinate of $\gamma$ as an element of $\mathbb{R}^{n}$, and $K$ is a Calderón-Zygmund kernel on the space $\Gamma^{\prime \prime} \cap \Gamma$. Thus the above theorem provides $L^{p}$ bounds for such operators provided $n-1 \leq 2 d$, where $d$ is the dimension of $\Gamma^{\prime \prime} \cap \Gamma$. This gives a partial answer to question (2) raised by Kenig and Stein in [20]. 
The non-degeneracy condition in the above theorem suggests —as in the case of the bilinear Hilbert transform - the question of uniform bounds as the subspace $\Gamma^{\prime}$ approaches the degenerate case. It turns out that for certain degenerate $\Gamma^{\prime}$ the form $\Lambda$ splits and one can use Hölder's inequality to obtain bounds in the degenerate case, for other degenerate $\Gamma^{\prime}$ the question is more subtle and not understood.

The case $k=1$ is the most tractable: the following seems true and is work in progress by Muscalu, Tao, and the author: Let $n \geq 3$ and $\Gamma \subset \mathbb{R}^{n}$ as before. Let $\Gamma^{\prime}$ be a subspace of $\Gamma$ spanned by $\left(\gamma_{1}, \ldots, \gamma_{n}\right) \in \mathbb{R}^{n}$. Assume $\gamma_{i} \neq 0$ for all $i$. Define distances

$$
\begin{aligned}
d(x, y) & =\sup _{i}\left|x_{i}-y_{i}\right| /\left|\gamma_{i}\right| \\
d\left(x, \Gamma^{\prime}\right) & =\inf _{y \in \Gamma^{\prime}} d(x, y) .
\end{aligned}
$$

Assume all derivatives of $m$ up to some finite order satisfy

$$
\left|\partial^{\alpha} m(\xi)\right| \leq \prod_{i=1}^{n}\left(\left|\gamma_{i}\right| d\left(x, \Gamma^{\prime}\right)\right)^{-\left|\alpha_{i}\right|}
$$

(if, say, $m$ is extended to be constant perpendicular to $\Gamma$ ). Then, uniformly in the choice of $\Gamma^{\prime}$,

$$
\Lambda\left(f_{1}, \ldots, f_{n}\right) \leq C \prod_{i=1}^{n}\left\|f_{i}\right\|_{p_{i}}
$$

as long as (all locally $L^{2}$ case):

$$
\sum_{i=1}^{n} \frac{1}{p_{i}}=1, \quad 2<p_{i}<\infty .
$$

One reason that the case $k>1$ is not understood is that there is no obvious analogue of the definition of distance.

We conclude this section with the remark that considering degenerate spaces $\Gamma^{\prime}$, so that $\Lambda$ splits into easier objects, one obtains interesting new phenomena if a multiplier is singular at two different such degenerate subspaces $\Gamma^{\prime}$. This will be part of the discussion in the chapter on eigenfunction expansions of Schrödinger operators.

\subsection{Further remarks.}

1) In $[\mathbf{2 4}]$, M. T. Lacey has shown the deep result that that the maximal truncations of the bilinear Hilbert transform:

$$
H_{\alpha}^{\max }(f, g)(x):=\sup _{\epsilon>0}\left|\int_{\mathbb{R} \backslash[-\epsilon, \epsilon]} f(x-t) g(x-\alpha t) \frac{1}{t} d t\right|
$$


as well as the maximal operator

$$
M(f, g)(x):=\sup _{\epsilon>0} \frac{1}{\epsilon} \int_{-\epsilon}^{\epsilon}|f(x-t) g(x-\alpha t)| \frac{1}{t} d t
$$

satisfy the same bounds as in Theorem 1.2, provided $\alpha$ is not degenerate. For the maximal operator this is nontrivial only if the exponent $p$ in that theorem is less than or equal 1 . The proof is a combination of the methods used in the proof of Theorem 1.2 and a lemma by Bourgain [2] on certain maximal averages.

2) As outlined before, the theory of modulation invariant singular integrals forces one to look at frequency bands of a function at all possible sizes and locations in frequency:

$$
f * \phi_{k, l}
$$

with $\widehat{\phi}_{k, l}(x)=\widehat{\phi}\left(2^{k} \xi-l\right)$ and $\widehat{\phi}$ is some smooth approximation of the characteristic function of $[0,1]$.

Due to the Heisenberg uncertainty principle, the above convolution operator acts essentially local on spatial intervals of length $2^{k}$. It is thus natural to consider wave packets of the form

$$
\phi_{k, n, l}=2^{-\frac{k}{2}} \phi\left(2^{-k} x-n\right) e^{2 \pi i 2^{-k} x l} .
$$

In fact, the $n$-linear forms considered before can typically be written as a sum of rank one tensors

$$
\sum_{P \in \mathbf{P}} c_{P}\left\langle f_{1}, \phi_{P, 1}\right\rangle \ldots\left\langle f_{n}, \phi_{P, n}\right\rangle
$$

which elucidates their intrinsic structure. Here $\mathbf{P}$ is simply some index set and $\phi_{1 P}, \ldots, \phi_{n, P}$ are wave packets.

Once written in this form, we may replace the wave packets $\phi_{P i}$ by Walsh wave packets $w_{P, i}$.

$$
\sum_{P \in \mathbf{P}} c_{P}\left\langle f_{1}, w_{P, 1}\right\rangle \ldots\left\langle f_{n}, w_{P, n}\right\rangle .
$$

Here a Walsh wave packet is defined as

$$
w_{k, n, l}=2^{-\frac{k}{2}} 1_{[0,1)}\left(2^{-k} x-n\right) \chi_{l}^{2 \pi i 2^{-k} x}
$$

where $\chi_{l}$ is the $l$-th character of the Walsh-Fourier transform, which is the abstract Fourier transform on the interval $[0,1)$ viewed as a group under bitwise addition of its elements written as binary numbers. See [38], [39] for details and original references.

While the original Fourier wave packet cannot be compactly supported if their Fourier transforms are compactly supported, the Walsh 
wave packets are compactly supported as well as their Walsh-Fourier transform. In fact the two supports are intervals and the product of their lengths is equal to 1: a sharp Heisenberg principle.

Virtually all results in the theory of modulation invariant operators have their Walsh-counterparts, which are technically much easier due to the exact space-frequency localization, but display much of the important structure of the result. To give an example of the technical simplifications in the Walsh model, consider a wave packet $\phi_{k, n, l}$. Its spacial support is mostly (in the Walsh case exactly) contained in the interval $\left[2^{k} n, 2^{k}(n+1)\right.$ ), whereas its frequency support (support of the Fourier transform) is contained in $\left[2^{-k} l, 2^{-k}(l+1)\right)$. We may draw the rectangle of the space-frequency localization:

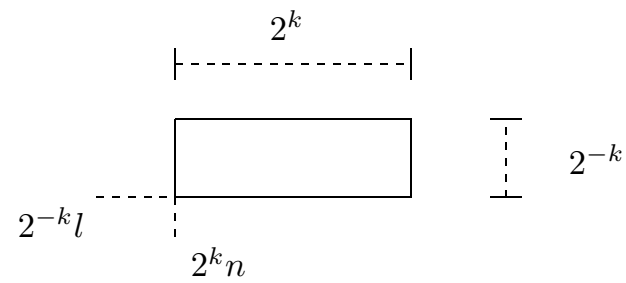

An essential tool in all boundedness results of modulation invariant singular integrals are estimates of the Bessel type

$$
\sum\left|\left\langle f, \phi_{j, p}\right\rangle\right|^{2} \leq C\|f\|_{2}^{2}
$$

provided the sum on the left hand side runs over a set of indices corresponding to pairwise disjoint rectangles:

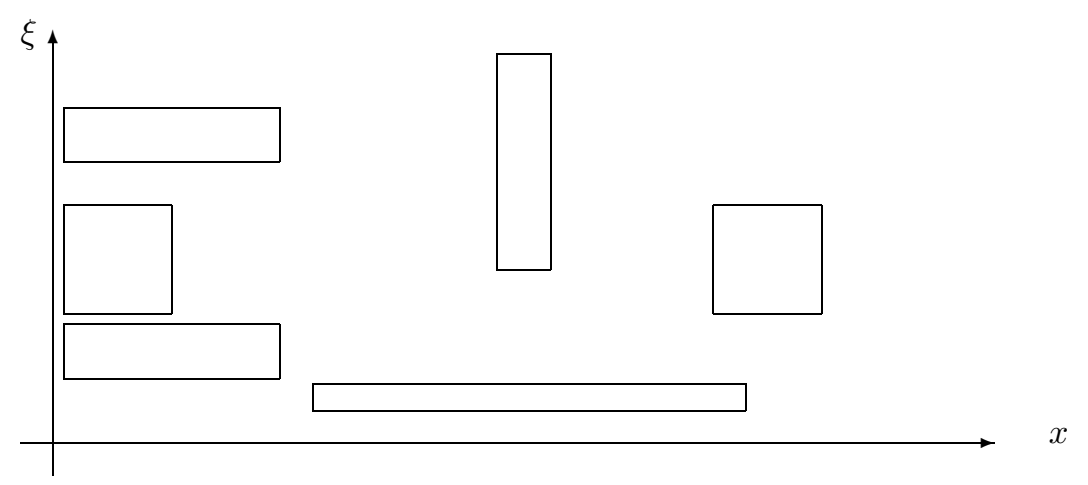


This Bessel inequality is true in the Walsh case, because the wave packets are pairwise orthogonal as one can see from either disjointness of spatial supports or disjointness in frequency supports and the Walsh Plancherel formula. In the Fourier case, Bessels inequality is not correct in general, not even with a large constant on the right hand side. Thus it has to be replaced by more technical inequalities, see for example [1].

\section{Boundedness of Carleson's operator}

\subsection{Introduction.}

As an illustration of the additional machinery needed to turn Littlewood-Paley theory into a useful tool in the modulation invariant setting, we give a proof of boundedness of Carleson's operator. We follow closely [29], with further comments as presented during the lecture at El Escorial. The Carleson operator $\mathcal{C}$ acting on a Schwartz function $f$ on $\mathbb{R}$ is defined by

$$
\mathcal{C} f(x):=\sup _{N}\left|\int_{-\infty}^{N} \widehat{f}(\xi) e^{2 \pi i \xi x} d \xi\right|,
$$

where the Fourier transform $\widehat{f}$ is defined by

$$
\widehat{f}(\xi):=\int f(x) e^{-2 \pi i \xi x} d x .
$$

We give a simplified proof of the well known theorem $[\mathbf{6}],[\mathbf{1 2}]$ :

Theorem 2.1. The Carleson operator $\mathcal{C}$ is of weak type $(2,2)$, i.e.,

$$
\|\mathcal{C} f\|_{L^{2, \infty}} \leq C\|f\|_{2}
$$

with a constant $C$ not depending on $f$.

This theorem is the key ingredient in the proof of Carleson's celebrated theorem, which asserts that the Fourier series of a function in $L^{2}([0,1])$ converges pointwise almost everywhere.

While the proof of L. Carleson [6] uses a decomposition of the function $f$ and the proof of C. Fefferman [12] uses a decomposition of the Carleson operator guided by $N$ (the function which picks the worst $N$ for each $x$ in the Carleson operator), we emphasize a symmetry between $f$ and $N$, as expressed by the duality of Propositions 2.2 and 2.3. These propositions are the key ingredients which make Littlewood Paley theory applicable in the modulation invariant setting. 
The similar role of these two proposition becomes even more evident in the case of the bilinear Hilbert transform (which we do not discuss here, see [25] and [27]), where instead of $f$ and $N$ one has three Schwartz functions $f_{1}, f_{2}$, and $f_{3}$, and a variant of Proposition 2.3 is applied to all three of them.

In Section 2.2 we discretize the Carleson operator. In Section 2.3 we prove boundedness of the discretized Carleson operator by taking for granted Propositions 2.2 and 2.3 and some technical inequality (20) from standard singular integral theory. These remaining items are proved in Sections 2.4, 2.5, and 2.6.

\subsection{Notation and preliminary reductions.}

Define translation, modulation, and dilation operators by

$$
\begin{aligned}
T_{y} f(x) & =f(x-y), \\
M_{\eta} f(x) & =f(x) e^{2 \pi i \eta x}, \\
D_{\Lambda}^{p} f(x) & =\Lambda^{-\frac{1}{p}} f\left(\Lambda^{-1} x\right) .
\end{aligned}
$$

Let $\phi$ be a Schwartz function such that $\widehat{\phi}$ is real, non-negative, supported in $[-0.1,0.1]$, and equal to 1 on $[-0.09,0.09]$.

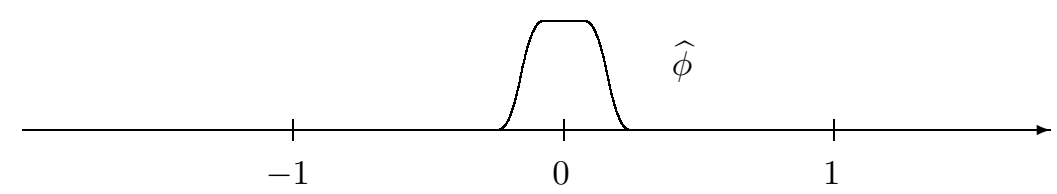

For each rectangle $P=I_{P} \times \omega_{P}$ of area 1 in the (phase-) plane define

$$
\phi_{1 P}:=M_{c\left(\omega_{1 P}\right)} T_{c\left(I_{P}\right)} D_{\left|I_{P}\right|}^{2} \phi,
$$

where we have written $\omega_{1 P}$ for the lower half of $\omega_{P}$ and $c(I)$ for the center of an interval $I$.

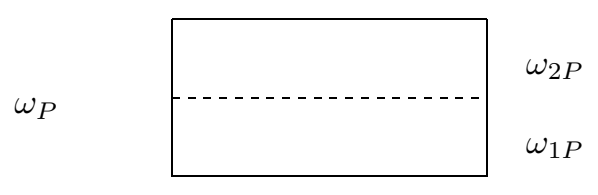

$I_{P}$ 
Observe that $\widehat{\phi}_{1 P}$ is supported in $\frac{1}{2} \omega_{1 P}$ (the interval of length $\left|\frac{1}{2} \omega_{1 P}\right|$ with the same center as $\omega_{1 P}$ ) and we have

$$
\left|\phi_{1 P}(x)\right| \leq C\left|I_{P}\right|^{\frac{1}{2}} w_{P}(x),
$$

where $w_{P}:=w_{I_{P}}$,

$$
w(x):=(1+|x|)^{-\nu}, \quad w_{I}(x):=T_{c(I)} D_{|I|}^{1} w .
$$

In this sense, the function $\phi_{1 P}$ is space-frequency-localized to the lower half of the rectange $I_{P} \times \omega_{P}$.

A dyadic interval is of the form $\left[n 2^{k},(n+1) 2^{k}\right)$ with integers $n$ and $k$. Let $\overline{\mathbf{P}}$ denote the set of rectangles $I \times \omega$ with $I, \omega$ dyadic and $|I||\omega|=1$. Define

$$
A_{\xi} f:=\sum_{P \in \overline{\mathbf{P}}} 1_{\omega_{2 P}}(\xi)\left\langle f, \phi_{1 P}\right\rangle \phi_{1 P}
$$

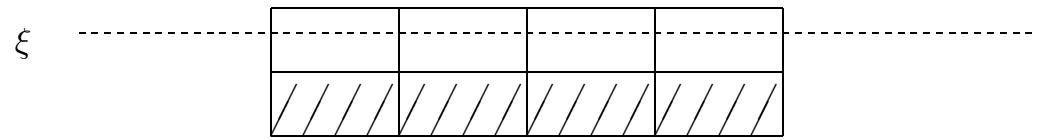

This is a non-zero, positive semi-definite operator and $A_{\xi} f$ vanishes if $\widehat{f}$ is supported in $[\xi, \infty]$. The role of each summand $P$ is to capture the space-frequency localized portion of $f$ that lives in space near $I_{P}$ and in frequency just underneath $\xi$; provided $\xi$ itself is contained in the upper half of $\omega_{P}$ :

Observe that the dyadic grid is invariant under dyadic dilations, hence for every integer $k$ we have

$$
A_{\xi} f=D_{2^{-k}}^{2} A_{2^{-k} \xi} D_{2^{k}}^{2} f .
$$

Averaging over translates and dilates of the involved dyadic structures we define

$$
\Pi_{\xi} f:=\lim _{n \rightarrow \infty} \frac{1}{\left|K_{n}\right|} \int_{K_{n} \times[0,1]} M_{-\eta} T_{-y} D_{2^{-\kappa}}^{2} A_{2^{-\kappa}(\eta+\xi)} D_{2^{\kappa}}^{2} T_{y} M_{\eta} f d y d \eta d \kappa,
$$

where $K_{n}$ is any increasing sequence of rectangles $I_{n} \times \omega_{n}$ filling out $\mathbb{R}^{2}$. To see the pointwise convergence of the last expression, consider separately those rectangles $P \in \overline{\mathbf{P}}$ with $\left|I_{P}\right|$ fixed, then the integrand becomes periodic in $y$ and $\eta$, and observe that for very large and very small values of $\left|I_{P}\right|$ the integrand becomes small. It is easy to verify that $\Pi_{\xi}$ extends 
to a bounded operator on $L^{2}$, is non-zero and positive semidefinite, commutes with $T_{y}$ for all $y$ and with $M_{\xi} D_{\lambda}^{2} M_{-\xi}$ for all $\lambda>0$, and satisfies $\Pi_{\xi} f=0$ if $\widehat{f}$ is supported in $[\xi, \infty]$. This identifies $\Pi_{\xi}$ as

$$
\Pi_{\xi} f(x)=c_{\xi} \int_{-\infty}^{\xi} \widehat{f}(\eta) e^{2 \pi i x \eta} d \eta
$$

for some constant $c_{\xi} \neq 0$. By conjugating with $M_{\xi^{\prime}}$ one observes that $c=c_{\xi}$ does not depend on $\xi$. Hence the Carleson operator is equal to $\mathcal{C} f(x)=c^{-1} \sup _{\xi}\left|\Pi_{\xi} f(x)\right|$. We will prove that

$$
\left\|\sup _{\xi}\left|A_{\xi} f\right|\right\|_{L^{2, \infty}} \leq C\|f\|_{2}
$$

By averaging this implies

$$
\begin{aligned}
& \left\|\sup _{\xi}\left|\Pi_{\xi} f(x)\right|\right\|_{2, \infty} \\
& \quad \leq \limsup _{n} \frac{1}{\left|K_{n}\right|} \int\left\|\sup _{\xi}\left|A_{\xi} D_{2^{\kappa}} T_{y} M_{\eta} f\right|\right\|_{2, \infty} d y d \eta d \kappa \leq C\|f\|_{2}
\end{aligned}
$$

which is enough to conclude Theorem 2.1.

By duality and the triangle inequality estimate (14) follows from

$$
\sum_{P \in \mathbf{P}}\left|\left\langle f, \phi_{1 P}\right\rangle\left\langle\phi_{1 P}\left(1_{\omega_{2 P}} \circ N\right), 1_{E}\right\rangle\right| \leq C\|f\|_{2}|E|^{\frac{1}{2}}
$$

for all Schwartz functions $f$, measurable functions $N$, measurable sets $E$, and finite subsets $\mathbf{P}$ of $\overline{\mathbf{P}}$. Since this estimate is homogeneous in $f$ and invariant under appropriate simultaneous dilations of $f, N, E$, and $\mathbf{P}$, it suffices to prove the estimate for $\|f\|_{2}=1$ and $|E| \leq 1$.

\subsection{The main argument.}

A rectangle $P=I_{P} \times \omega_{P}$ of $\overline{\mathbf{P}}$ will be called a tile. Each tile has area 1 and is the union of two semitiles $P_{1}=I_{P} \times \omega_{1 P}$ and $P_{2}=I_{P} \times \omega_{2 P}$. Observe that dyadic intervals such as $I_{P}, \omega_{P}, \omega_{j P}$ have the property that any two of them are either disjoint or one is contained in the other. Moreover, if $\omega_{1 P}$ is strictly contained in a dyadic interval, then $\omega_{2 P}$ is strictly contained in the same interval and vice versa. We will use these geometric properties without referring to them. 
We define a partial ordering on the set of tiles by $P<P^{\prime}$ if $I_{P} \subset I_{P^{\prime}}$ and $\omega_{P^{\prime}} \subset \omega_{P}$.

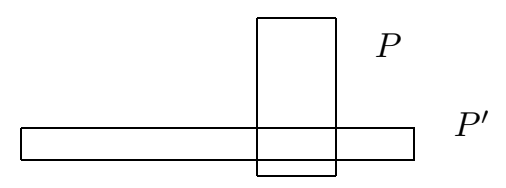

If $P<P^{\prime}$ we have two types of intersection: $P^{\prime}$ intersects either the lower half or the upper half of $P$.

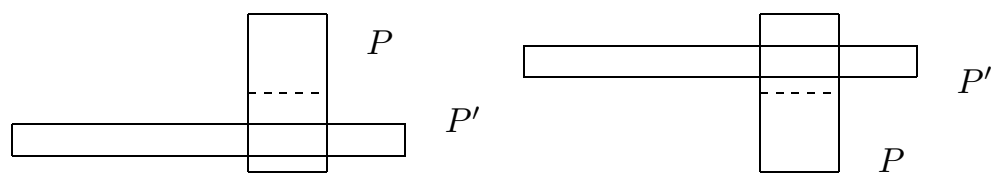

A set $T$ of tiles is called a tree, if there is a tile $P_{T}=I_{T} \times \omega_{T}$, the top of the tree, such that $P<P_{T}$ for all $P \in T$. Observe that we do not require the top to be an element of the tree.

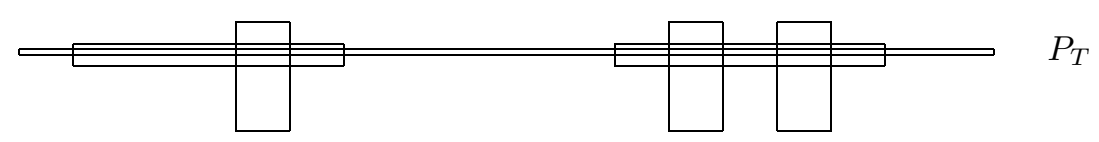

A tree is called $j$-tree if $\omega_{j P_{T}} \subset \omega_{j P}$ for all $P \in T$. The following is a 2-tree:

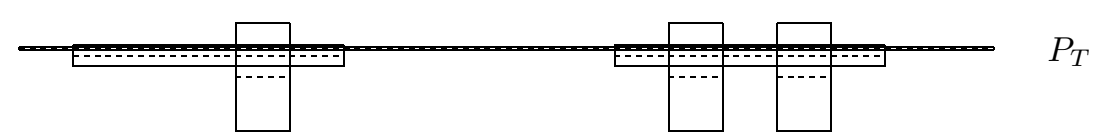

We will introduce quantities mass and energy which measure the behaviour and distribution of the linearizing function $N$ and the function $f$ respectively. 
Recall that we are given a fixed set $E$ with measure $|E| \leq 1$. Define $E_{P}:=E \cap\left\{x: N(x) \in \omega_{P}\right\}, \quad E_{2 P}:=E \cap\left\{x: N(x) \in \omega_{2 P}\right\}$.

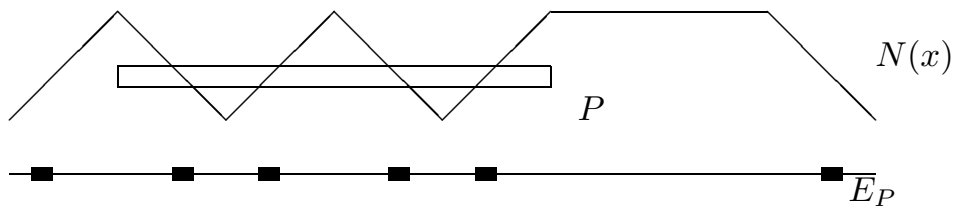

For a finite subset $\mathbf{P} \subset \overline{\mathbf{P}}$, define

$$
\operatorname{mass}(\mathbf{P}):=\sup _{P \in \mathbf{P}} \sup _{P^{\prime} \in \overline{\mathbf{P}}: P<P^{\prime}} \int_{E_{P^{\prime}}} w_{P^{\prime}}(x) d x,
$$

where $w_{P^{\prime}}$ is the weight function as in (13). The integral in this definition captures the density of $E_{P}^{\prime}$ near $I_{P^{\prime}}$.

The following proposition is a way of saying that there is only a limited amount of mass in the phase plane, so only a limited amount of tiles can carry large mass:

Proposition 2.2. Let $\mathbf{P}$ be a finite set of tiles, then $\mathbf{P}$ can be decomposed as the union of sets $\mathbf{P}_{\text {light }}$ and $\mathbf{P}_{\text {heavy }}$ with

$$
\operatorname{mass}\left(\mathbf{P}_{\text {light }}\right) \leq 2^{-1} \operatorname{mass}(\mathbf{P}),
$$

and $\mathbf{P}_{\text {heavy }}$ is the union of a set $\mathbf{T}$ of trees such that

$$
\sum_{T \in \mathbf{T}}\left|I_{T}\right| \leq C \operatorname{mass}(\mathbf{P})^{-1} .
$$

Now recall that we are given a function $f$ with $\|f\|_{2} \leq 1$. For a finite subset $\mathbf{P} \subset \overline{\mathbf{P}}$, define

$$
\operatorname{energy}(\mathbf{P}):=\sup _{T}\left(\left|I_{T}\right|^{-1} \sum_{P \in T}\left|\left\langle f, \phi_{1 P}\right\rangle\right|^{2}\right)^{\frac{1}{2}},
$$

where the sup is taken over all 2-trees $T \subset \mathbf{P}$.

Observe that the lower half boxes of a 2-tree, which carry the localized functions $\phi_{1 P}$, are pairwise disjoint.

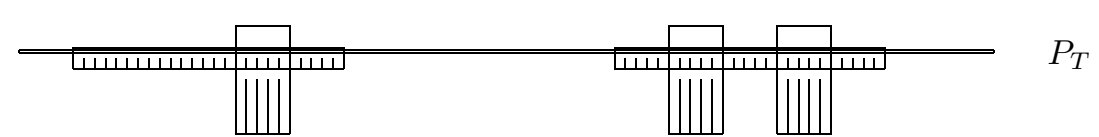

The following proposition says that there is only a limited amount of energy distributed over the phase-plane. 
Proposition 2.3. Let $\mathbf{P}$ be a finite set of tiles, then $\mathbf{P}$ can be decomposed as the union of $\mathbf{P}_{\text {low }}$ and $\mathbf{P}_{\text {high }}$ with

$$
\operatorname{energy}\left(\mathbf{P}_{\text {low }}\right) \leq 2^{-1} \operatorname{energy}(\mathbf{P}),
$$

and $\mathbf{P}_{\text {high }}$ is the union of a set $\mathbf{T}$ of trees such that

$$
\sum_{T \in \mathbf{T}}\left|I_{T}\right| \leq C \operatorname{energy}(\mathbf{P})^{-2}
$$

We prove these propositions in Sections 2.4 and 2.5. Assuming these propositions are correct, and assuming the validity of a technical estimate (20) below, we can now quickly finish the proof of Theorem 2.1.

Namely, given a finite collection $\mathbf{P}$ of tiles, we use Propositions 2.2 and 2.3 to obtain a decomposition of $\mathbf{P}$ into sets $\mathbf{P}_{n}$, where $n$ runs through some finite set of integers, such that for each $n$ we have

$$
\operatorname{mass}\left(\mathbf{P}_{n}\right) \leq 2^{2 n}, \quad \operatorname{energy}\left(\mathbf{P}_{n}\right) \leq 2^{n}
$$

and $\mathbf{P}_{n}$ is the union of a set $\mathbf{T}_{n}$ of trees with

$$
\sum_{T \in \mathbf{T}_{n}}\left|I_{T}\right| \leq C 2^{-2 n}
$$

Namely, initially $\mathbf{P}$ satisfies estimates as in (18) for some large $n$. If the mass of $\mathbf{P}$ is greater than $2^{2(n-1)}$, we split it into $\mathbf{P}_{\text {light }}$ and $\mathbf{P}_{\text {heavy }}$, replace $\mathbf{P}$ by $\mathbf{P}_{\text {light }}$, and add $\mathbf{P}_{\text {heavy }}$ to $\mathbf{P}_{n}$. Then, if the energy of $\mathbf{P}$ is greater than $2^{n-1}$, we split $\mathbf{P}$ into $\mathbf{P}_{\text {high }}$ and $\mathbf{P}_{\text {low }}$, replace $\mathbf{P}$ by $\mathbf{P}_{\text {low }}$, and add $\mathbf{P}_{\text {high }}$ to $\mathbf{P}_{n}$. Then $\mathbf{P}$ satisfies (18) with $n$ replaced by $n-1$ and we iterate.

In Section 2.6 we prove for each tree $T$ the - unfortunately a bit technical but otherwise rather standard - inequality in Calderón-Zygmund theory

$$
\sum_{P \in T}\left|\left\langle f, \phi_{1 P}\right\rangle\left\langle\phi_{1 P}, 1_{E_{2 P}}\right\rangle\right| \leq C \operatorname{energy}(T) \operatorname{mass}(T)\left|I_{T}\right| .
$$

Hence, with the observation that the mass of any collection of tiles is bounded by a universal constant, we have the estimate

$$
\sum_{T \in \mathbf{T}_{n}} \sum_{P \in T}\left|\left\langle f, \phi_{1 P}\right\rangle\left\langle\phi_{1 P}, 1_{E_{2 P}}\right\rangle\right| \leq C 2^{n} \min \left(C, 2^{2 n}\right) 2^{-2 n} .
$$

This is summable over $n \in \mathbb{Z}$, which proves (15) and therefore Theorem 2.1 .

\subsection{Proof of Proposition 2.2.}

To motivate things, we first prove Proposition 2.2 for a slightly easier notion of mass, where the weight function $w$ is replaced by a sharp cutoff function. 
For $\mathbf{P}$ a set of tiles define

$$
\operatorname{mass}^{\prime}(\mathbf{P}):=\max _{P \in \mathbf{P}, P^{\prime} \in \overline{\mathbf{P}}: P<P^{\prime}}\left|I_{P^{\prime}} \cap E_{P^{\prime}}\right| /\left|I_{P^{\prime}}\right| .
$$

Let $\mathbf{P}_{2}$ be the set of $P \in \mathbf{P}$ with

$$
\operatorname{mass}^{\prime}(\{P\}) \geq \operatorname{mass}^{\prime}(\mathbf{P}) / 2 \text {. }
$$

Then $\mathbf{P}_{1}=\mathbf{P} \backslash \mathbf{P}_{2}$ is as required in Proposition 2.2.

Let $\mathbf{P}^{\prime}$ be the set of maximal tiles $P^{\prime}$ in $\overline{\mathbf{P}}$ with respect to the tile ordering for which:

$$
\left|I_{P^{\prime}} \cap E_{P^{\prime}}\right| /\left|I_{P^{\prime}}\right| \geq \operatorname{mass}^{\prime}(\mathbf{P}) / 2 .
$$

Then, trivially, $\mathbf{P}_{2}$ is a union of trees $T$ with $P_{T} \in P^{\prime}$.

The tiles $P^{\prime} \in \mathbf{P}^{\prime}$ are pairwise disjoint because they are pairwise incomparable w.r.t. the tile ordering. Hence the sets $I_{P^{\prime}} \cap E_{P^{\prime}}$, are pairwise disjoint, as is illustrated by following figure:

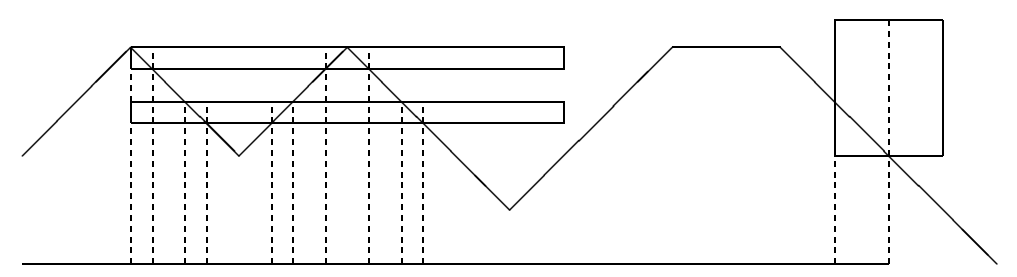

Hence we have

$$
\sum_{P^{\prime} \in \mathbf{P}^{\prime}}\left|I_{P}\right| \leq \sum_{P^{\prime} \in \mathbf{P}^{\prime}} \frac{2\left|I_{P^{\prime}} \cap E_{P^{\prime}}\right|}{\operatorname{mass}^{\prime}(\mathbf{P})} \leq \frac{2}{\operatorname{mass}^{\prime}(\mathbf{P})},
$$

which proves Proposition 2.2 for mass'.

Now we pass to the proof of Proposition 2.2 for the original notion of mass. Since the weight function $w$ allows for a small far field contribution to the mass of a tile, we have to consider a dyadic sequence of auxiliary rectangles and apply the old argument to these rectangles:

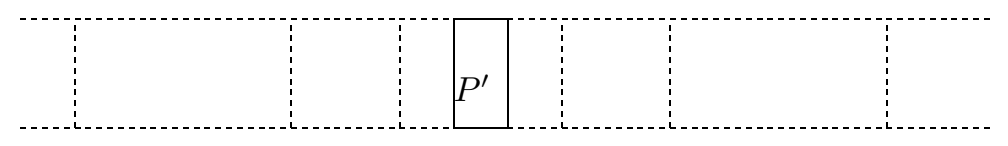

Let $\mu=\operatorname{mass}(\mathbf{P})$. Let $\mathbf{P}^{\prime}$ be those elements $P^{\prime}$ in $\overline{\mathbf{P}}$ with

$$
\int_{E_{P^{\prime}}} w_{P^{\prime}} d x>2^{-1} \mu
$$


which are maximal with respect to the partial order $<$ of tiles. It suffices to show that

$$
\sum_{P^{\prime} \in \mathbf{P}^{\prime}}\left|I_{P^{\prime}}\right|<C \mu^{-1},
$$

because the tiles $P \in \mathbf{P}_{\text {heavy }}$ can be collected into trees with tops in $\mathbf{P}^{\prime}$.

For $\kappa \in \mathbb{N}_{0}$ define $\mathbf{P}_{\kappa}$ to be the set of all $P \in \mathbf{P}^{\prime}$ with

$$
\left|E_{P} \cap 2^{\kappa} I_{P}\right| \geq c 2^{2 \kappa} \mu\left|I_{P}\right|
$$

for some constant $c$. If $c$ is small enough, then one can conclude from the mass estimate that each element $P$ of $\mathbf{P}^{\prime}$ is contained in one of the sets $\mathbf{P}_{\kappa}$. Hence it suffices to show for every $\kappa$

$$
\sum_{P \in \mathbf{P}_{\kappa}}\left|I_{P}\right| \leq C 2^{-\kappa} \mu^{-1} .
$$

Fix $\kappa$. For each $P \in \mathbf{P}_{\kappa}$ we have an enlarged rectangle $\left(2^{\kappa} I_{P}\right) \times \omega_{P}$. We select successively elements $P \in \mathbf{P}_{\kappa}$ with maximal $\left|I_{P}\right|$ whose enlarged rectangles are disjoint from the enlarged rectangles of all previously selected elements. When no further element can be selected, then each rectangle $P^{\prime} \in \mathbf{P}_{\kappa}$ can be associated to a selected rectangle $P$ such that $\left|I_{P^{\prime}}\right|<\left|I_{P}\right|$ and the enlarged rectangles of $P$ and $P^{\prime}$ intersect. Since the rectangles in $\mathbf{P}_{\kappa}$ are pairwise disjoint, we see that the intervals $I_{P^{\prime}}$ of the rectangles $P^{\prime}$ associated to a fixed selected $P$ are pairwise disjoint and contained in $2^{\kappa+2} I_{P}$. Hence

$$
\begin{aligned}
\sum_{P \in \mathbf{P}_{\kappa}}\left|I_{P}\right| \leq C 2^{\kappa} \sum_{P \text { selected }}\left|I_{P}\right| & \leq C 2^{-\kappa} \mu^{-1} \sum_{P \text { selected }}\left|E_{P} \cap 2^{\kappa} I_{P}\right| .
\end{aligned}
$$

This is bounded by $C 2^{-\kappa} \mu^{-1}$ because the enlarged rectangles of the selected elements $P$ are pairwise disjoint and therefore the subsets $E_{P} \cap$ $2^{\kappa} I_{P}$ of $E$ are pairwise disjoint. This finishes the proof of Proposition 2.2.

\subsection{Proof of Proposition 2.3.}

Let $\varepsilon=\operatorname{energy}(\mathbf{P})$. For a 2 -tree $T$, let

$$
\Delta(T)^{2}=\left|I_{T}\right|^{-1} \sum_{P \in T}\left|\left\langle f, \phi_{1 P}\right\rangle\right|^{2} .
$$

We inductively construct the collection $\mathbf{T}$ of trees whose union will be $\mathbf{P}_{\text {high }}$.

Pick a 2-tree $T \in \mathbf{P}$ such that (1) $\Delta(T) \geq 2^{-1} \varepsilon$ and (2) $c\left(\omega_{T}\right)$ is minimal among all 2-trees satisfying the first condition. Then let $T^{1}$ be the maximal (with respect to set inclusion) tree in $\mathbf{P}$ with top $I_{T} \times \omega_{T}$.

Add $T^{1}$ to $\mathbf{T}$, add $T$ to $\mathbf{T}_{2}$, which will be a collection of 2 -trees we will work with in the sequel because it has better disjointness properties 
than the collection $\mathbf{T}$. Remove each element of $T^{1}$ from $\mathbf{P}$. Then repeat the procedure above until there is no tree in $\mathbf{P}$ with $\Delta(T) \geq 2^{-1} \varepsilon$. Then we can define $\mathbf{P}$ to be $\mathbf{P}_{\text {light }}$.

The main disjointness property is the following: Let $T, T^{\prime} \in \mathbf{T}_{2}$ and let $P \in T$ and $P^{\prime} \in T^{\prime}$. Then we have: if $\omega_{P}$ is contained in $\omega_{1 P^{\prime}}$, then $I_{P^{\prime}} \cap I_{T}=\emptyset$. To see this, note that $c\left(\omega_{T}\right)$, which is contained in $\omega_{P}$, is less than $c\left(\omega_{T^{\prime}}\right) \in \omega_{2 P^{\prime}}$. Therefore $T$ was selected before $T^{\prime}$. But if $I_{P^{\prime}}$ and $I_{T}$ had nonempty intersection, then $P^{\prime}$ would qualify to be in the tree $T^{1}$ and would have been removed from $\mathbf{P}$ before $T^{\prime}$ was selected. This is impossible.

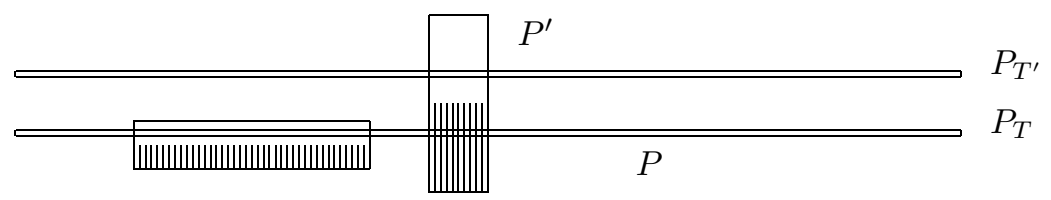

In particular if $P \in T$ and $P \in T^{\prime}$ with $T, T^{\prime} \in \mathbf{T}_{2}$, then the semitiles $P_{1}$ and $P_{1}^{\prime}$ are disjoint.

To motivate things, assume for a moment that the wave packets $\phi_{1 P}$ and $\phi_{1 P^{\prime}}$ were orthogonal, as is suggested by the above disjointness property. Then we had by Bessel's inequality

$$
\sum_{T \in \mathbf{T}}\left|I_{P_{T}}\right|=\sum_{T \in \mathbf{T}_{2}}\left|I_{P_{T}}\right| \leq 4 \varepsilon^{-2} \sum_{T \in \mathbf{T}_{2}} \sum_{P \in T}\left|\left\langle f, \phi_{1 P}\right\rangle\right|^{2} \leq 4 \varepsilon^{-2},
$$

which would prove Proposition 2.3.

However, the functions $\phi_{1 P}$ and $\phi_{1 P^{\prime}}$ are not quite orthogonal, so we need a more refined argument.

We are aiming to show that

$$
\varepsilon^{2} \sum_{T \in \mathbf{T}_{2}}\left|I_{T}\right| \leq C
$$

Letting $\overline{\mathbf{P}}$ be the union of the 2-trees $T$ in $\mathbf{T}_{2}$, the left hand side is at most a constant times

$$
\sum_{P \in \overline{\mathbf{P}}}\left|\left\langle f, \phi_{1 P}\right\rangle\right|^{2} \leq\left\|\sum_{P \in \overline{\mathbf{P}}}\left\langle f, \phi_{1 P}\right\rangle \phi_{1 P}\right\|_{2}^{2} .
$$

Here we have used that the $L^{2}$-norm of $f$ is 1 .

Therefore, it is sufficient to prove

$$
\left\|\sum_{P \in \overline{\mathbf{P}}}\left\langle f, \phi_{1 P}\right\rangle \phi_{1 P}\right\|_{2}^{2} \leq C \varepsilon^{2} \sum_{T \in \mathbf{T}_{2}}\left|I_{T}\right| .
$$


We estimate the left hand side of (21) by

$$
\begin{array}{r}
\sum_{P, P^{\prime} \in \overline{\mathbf{P}}: \omega_{P}=\omega_{P^{\prime}}}\left|\left\langle f, \phi_{1 P}\right\rangle\left\langle\phi_{1 P}, \phi_{1 P^{\prime}}\right\rangle\left\langle\phi_{1 P^{\prime}}, f\right\rangle\right| \\
+2 \sum_{P, P^{\prime} \in \overline{\mathbf{P}}: \omega_{P} \subset \omega_{1 P^{\prime}}}\left|\left\langle f, \phi_{1 P}\right\rangle\left\langle\phi_{1 P}, \phi_{1 P^{\prime}}\right\rangle\left\langle\phi_{1 P^{\prime}}, f\right\rangle\right| .
\end{array}
$$

Here we have used symmetry and the fact that $\left\langle f, \phi_{1 P}\right\rangle=0$ unless one of the intervals $\omega_{1 P}$ and $\omega_{1 P^{\prime}}$ is contained in the other.

Observe that for $\left|I_{P^{\prime}}\right| \leq\left|I_{P}\right|$ we have

$$
\left|\left\langle\phi_{1 P}, \phi_{1 P^{\prime}}\right\rangle\right| \leq C\left|I_{P}\right|^{\frac{1}{2}}\left|I_{P^{\prime}}\right|^{-\frac{1}{2}}\left\|w_{P} 1_{I_{P^{\prime}}}\right\|_{1} .
$$

We estimate the smaller one of $\left|\left\langle f, \phi_{1 P}\right\rangle\right|$ and $\left|\left\langle f, \phi_{1 P^{\prime}}\right\rangle\right|$ by the larger one and use symmetry to obtain for (22) the upper bound

$$
C \sum_{P \in \overline{\mathbf{P}}}\left|\left\langle f, \phi_{1 P}\right\rangle\right|^{2} \sum_{P^{\prime} \in \overline{\mathbf{P}}: \omega_{P}=\omega_{P^{\prime}}}\left\|w_{P} 1_{I_{P^{\prime}}}\right\|_{1} .
$$

We can estimate the interior sum by $\left|I_{P}\right|^{-1}\left\|w_{P}\right\|_{1} \leq C$, because the intervals $I_{P^{\prime}}$ with $\omega_{P^{\prime}}=\omega_{P}$ are pairwise disjoint. This proves the desired bound for $(22)$.

We can estimate the second summand (23) by

$$
\begin{aligned}
& \sum_{P \in \overline{\mathbf{P}}}\left|\left\langle f, \phi_{1 P}\right\rangle\right| \sum_{P^{\prime} \in \overline{\mathbf{P}}: \omega_{P} \subset \omega_{1 P^{\prime}}}\left|\left\langle\phi_{1 P}, \phi_{1 P^{\prime}}\right\rangle\left\langle\phi_{1 P^{\prime}}, f\right\rangle\right| \\
& \leq \sum_{T \in \mathbf{T}_{2}}\left(\sum_{P \in T}\left|\left\langle f, \phi_{1 P}\right\rangle\right|^{2}\right)^{\frac{1}{2}} H(T)^{\frac{1}{2}} \leq C \varepsilon \sum_{T \in \mathbf{T}_{2}}\left|I_{T}\right|^{\frac{1}{2}} H(T)^{\frac{1}{2}}
\end{aligned}
$$

where

$$
H(T):=\sum_{P \in T}\left(\sum_{P^{\prime} \in \overline{\mathbf{P}}: \omega_{P} \subset \omega_{1 P^{\prime}}}\left|\left\langle\phi_{1 P}, \phi_{1 P^{\prime}}\right\rangle\left\langle\phi_{1 P^{\prime}}, f\right\rangle\right|\right)^{2}
$$


The following figure shows an example for the tree $T$ and the set of $P^{\prime}$ for which $\omega_{P} \subset \omega_{1 P^{\prime}}$. Observe that the latter requires the intervals $I_{P^{\prime}}$ to be pairwise disjoint.

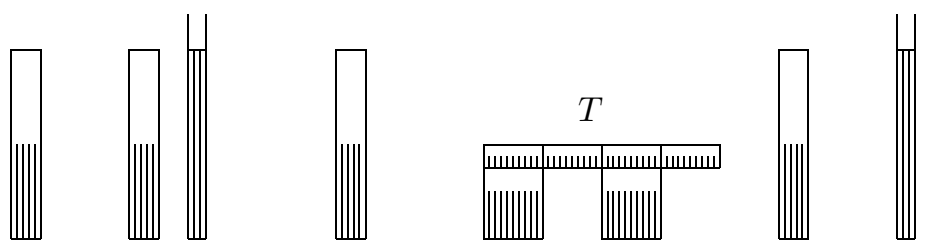

It remains to show that $H(T) \leq C \varepsilon^{2}\left|I_{T}\right|$ for each tree $T \in \mathbf{T}_{2}$.

But,

$$
H(T) \leq C \varepsilon^{2} \sum_{P \in T}\left|I_{P}\right|\left(\sum_{P^{\prime} \in \overline{\mathbf{P}}: \omega_{P} \subset \omega_{1 P^{\prime}}}\left\|w_{P} 1_{I_{P^{\prime}}}\right\|_{1}\right)^{2},
$$

where we have used the upper energy estimate for each individual $P^{\prime}$ (which is a 2-tree by itself), and the estimate on $\left\langle\phi_{1 P}, \phi_{1 P^{\prime}}\right\rangle$. Fix $P$, then the intervals $I_{P^{\prime}}$ with $\omega_{P} \subset \omega_{1 P^{\prime}}$ are pairwise disjoint and disjoint from $I_{T}$ by the above mentioned disjointness property. Hence we have

$$
\sum_{P^{\prime} \in \overline{\mathbf{P}}: \omega_{P} \subset \omega_{1 P^{\prime}}}\left\|w_{P} 1_{I_{P^{\prime}}}\right\|_{1} \leq C\left\|w_{P} 1_{I_{T}^{c}}\right\|_{1} .
$$

For each $x \in I_{T}$ there is at most one $P \in T$ of each scale with $x \in I_{P}$. Hence we have:

$$
\begin{aligned}
\sum_{P \in T}\left|I_{P}\right|\left\|w_{P} 1_{I_{T}^{c}}\right\|_{1}^{2} \leq C & \sum_{P \in T}\left|I_{P}\right|\left\|w_{P} 1_{I_{T}^{c}}\right\|_{1} \\
& \leq C \sum_{k \in \mathbb{N}}\left\|\left(1_{I_{T}} * D_{2^{-k}\left|I_{T}\right|}^{1} w\right) 1_{I_{T}^{c}}\right\|_{1} \leq C\left|I_{T}\right| .
\end{aligned}
$$

This gives the appropriate bound for $H(T)$ and thus finishes the proof of (21).

\subsection{Proof of estimate (20).}

From the point of view of classical Calderón-Zygmund theory, estimate (20) is a rather standard estimate for maximal Calderón-Zygmund operators, with a slight twist to obtain the factor $\mu$ in front.

Let $\mathcal{J}$ be the collection of all maximal dyadic intervals such that $3 J$ does not contain any $I_{P}$ with $P \in T$. Then $\mathcal{J}$ is a partition of $\mathbb{R}$ as shown in the following figure: 


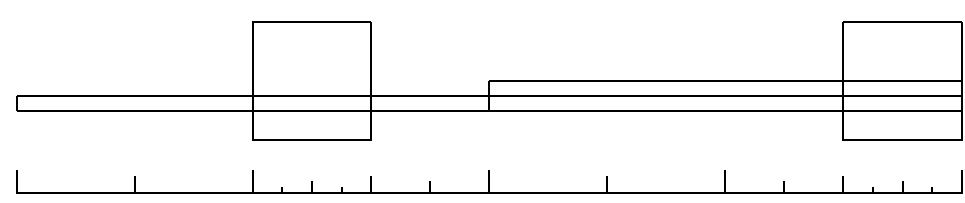

The main idea to obtain the extra factor $\mu$ is that the functions involved will live on sparse sets of density $\mu$ on each of the intervals in $\mathcal{J}$.

We estimate the left hand side of (20) as below, where the terms $\epsilon_{P}$ are phase factors of modulus 1 which make up for the absolute value signs in (20).

$$
\begin{aligned}
& \left\|\sum_{P \in T} \epsilon_{P}\left\langle f, \phi_{1 P}\right\rangle \phi_{1 P} 1_{E_{2 P}}\right\|_{1} \\
\leq & \sum_{J \in \mathcal{J}} \sum_{P \in T:\left|I_{P}\right| \leq|J|}\left\|\left\langle f, \phi_{1 P}\right\rangle \phi_{1 P} 1_{E_{2 P}}\right\|_{L^{1}(J)} \\
& +\sum_{J \in \mathcal{J}}\left\|\sum_{P \in T:\left|I_{P}\right|>|J|} \epsilon_{P}\left\langle f, \phi_{1 P}\right\rangle \phi_{1 P} 1_{E_{2 P}}\right\|_{L^{1}(J)} .
\end{aligned}
$$

To estimate (24), we calculate for each $J \in \mathcal{J}$ and $P \in T$ with $\left|I_{P}\right| \leq|J|$ :

$$
\left\|\left\langle f, \phi_{1 P}\right\rangle \phi_{1 P} 1_{E_{2 P}}\right\|_{L^{1}(J)} \leq C \varepsilon \mu\left|I_{P}\right|\left(1+\operatorname{dist}\left(I_{P}, J\right)\left|I_{P}\right|^{-1}\right)^{-\nu} .
$$

Here, we have set as before $\varepsilon:=\operatorname{energy}(T)$ and $\mu:=\operatorname{mass}(T)$. Fix an integer $k$ with $2^{k} \leq|J|$. Consider all $P \in T$ with $\left|I_{P}\right|=2^{k}$, then the intervals $I_{P}$ are pairwise disjoint, disjoint from $J$, and contained in $I_{T}$. Hence

$$
\sum_{P \in T:\left|I_{P}\right|=2^{k}}\left|I_{P}\right|\left(1+\operatorname{dist}\left(I_{P}, J\right)\left|I_{P}\right|^{-1}\right)^{-\nu} \leq C 2^{k}\left(1+\operatorname{dist}\left(I_{T}, J\right)\left|I_{T}\right|^{-1}\right)^{-\nu^{\prime}} .
$$

These estimates, summed over $2^{k} \leq|J|$ and $J \in \mathcal{J}$, yield no more than $C\left|I_{T}\right|$. Together with (26) this gives the desired bound for (24).

We consider (25). We can assume that the summation runs only over those $J \in \mathcal{J}$ for which there exists a $P \in T$ with $|J|<\left|I_{P}\right|$. Then we have $J \subset 3 I_{T}$ and $|J|<\left|I_{T}\right|$ for all $J$ occurring in the sum.

Fix an interval $J \in \mathcal{J}$ and observe that

$$
G_{J}:=J \cap \bigcup_{P \in T:\left|I_{P}\right|>|J|} E_{2 P}
$$

has measure at most $C \mu|J|$. Indeed, let $J^{\prime}$ be the dyadic interval which contains $J$ and $\left|J^{\prime}\right|=2|J| \leq\left|I_{T}\right|$. By maximality of $J, 3 J^{\prime}$ contains an 
interval $I_{P}$ for some $P \in T$. Let $P^{\prime}$ be the tile with $\left|I_{P^{\prime}}\right|=\left|J^{\prime}\right|$ and $P<P^{\prime}<I_{T} \times \omega_{T}$. Then $G_{J} \subset J \cap E_{P^{\prime}}$. And since $\operatorname{mass}(\{P\}) \leq \mu$, our claim follows.

Let $T_{2}$ be the 2-tree of all $P \in T$ such that $\omega_{2 T} \subset \omega_{2 P}$, and let $T_{1}=T \backslash T_{2}$. Define, for $j=1,2$,

$$
F_{j J}:=\sum_{P \in T_{j}:\left|I_{P}\right|>|J|} \epsilon_{P}\left\langle f, \phi_{1 P}\right\rangle \phi_{1 P} 1_{E_{2 P}} .
$$

If $P, P^{\prime}$ are in the 1-tree $T_{1}$ and have different scales, then the sets $\omega_{2 P}, \omega_{2 P^{\prime}}$ are disjoint and so are the sets $E_{2 P}, E_{2 P^{\prime}}$. Therefore, by considering single scales separately one can estimate the $L^{\infty}$ norm of $F_{1 J}$ by $C \epsilon$. Hence

$$
\left\|F_{1 J}\right\|_{L^{1}(J)} \leq C \varepsilon\left|G_{J}\right| \leq C \varepsilon \mu|J| .
$$

Summing over the disjoint intervals $J \subset 3 I_{T}$ gives the desired estimate for the $T_{1}$-part of (25).

Fix $x$ and assume that $F_{2 J}(x)$ is not zero. Since the intervals $\omega_{2 P}$ with $P \in T_{2}$ are nested, there is a largest (smallest) interval $\omega_{+}\left(\omega_{-}\right)$ of the form $\omega_{P}\left(\omega_{2 P}\right)$ with $P \in T_{2}, x \in E_{2 P}$ and $\left|I_{P}\right|>|J|$. In other words, we have for any $P \in T$ that $x \in E_{2 P}$ and $\left|I_{P}\right|>|J|$ if and only if $\left|\omega_{-}\right|<\left|\omega_{P}\right| \leq\left|\omega_{+}\right|$.

Hence we can write $F_{2 J}(x)$ as

$$
\begin{aligned}
& \sum_{P \in T_{2}:\left|\omega_{-}\right|<\left|\omega_{P}\right| \leq\left|\omega_{+}\right|} \epsilon_{P}\left\langle f, \phi_{1 P}\right\rangle \phi_{1 P}(x) \\
= & \sum_{P \in T_{2}} \epsilon_{P}\left\langle f, \phi_{1 P}\right\rangle\left(\phi_{1 P} *\left(M_{c\left(\omega_{+}\right)} D_{0.1\left|\omega_{+}\right|^{-1}}^{1} \phi-M_{c\left(\omega_{-}\right)} D_{0.09\left|\omega_{-}\right|^{-1}}^{1} \phi\right)\right)(x) .
\end{aligned}
$$

The last equality is easily seen from the geometry of the supports of the functions $\widehat{\phi}_{1 P}$. Therefore we can estimate $\left|F_{2 J}(x)\right|$ by

$$
C \sup _{J \subset I} \frac{1}{|I|} \int_{I}\left|\sum_{P \in T_{2}} \epsilon_{P}\left\langle f, \phi_{1 P}\right\rangle \phi_{1 P}(z)\right| d z,
$$

which is constant on $J$. 
But $F_{2 J} 1_{J}$ is supported on the set $G_{J}$ of measure $\leq C \mu|J|$, hence

$$
\begin{aligned}
& \sum_{J \in \mathcal{J}: J \subset 3 I_{T}}\left\|F_{2 J}\right\|_{L^{1}(J)} \\
\leq C & \sum_{J \in \mathcal{J}: J \subset 3 I_{T}} \mu|J| \sup _{J \subset I} \frac{1}{|I|} \int_{I}\left|\sum_{P \in T_{2}} \epsilon_{P}\left\langle f, \phi_{1 P}\right\rangle \phi_{1 P}(z)\right| d z \\
\leq & C \mu\left\|M\left(\sum_{P \in T_{2}} \epsilon_{P}\left\langle f, \phi_{1 P}\right\rangle \phi_{1 P}\right)\right\|_{L^{1}\left(3 I_{T}\right)} \\
\leq & C \mu\left|I_{T}\right|^{\frac{1}{2}}\left\|\sum_{P \in T_{2}} \epsilon_{P}\left\langle f, \phi_{1 P}\right\rangle \phi_{1 P}\right\|_{2} .
\end{aligned}
$$

Here $M$ denotes the Hardy Littlewood maximal function and we have used the maximal theorem.

We observe that for different scales of $P, P^{\prime} \in T_{2}$ the intervals $\omega_{1 P}$ and $\omega_{1 P^{\prime}}$ are disjoint and therefore the functions $\phi_{1 P}$ and $\phi_{1 P^{\prime}}$ are orthogonal. Thus, following the arguments to estimate (22), we estimate the $L^{2}$ norm in the last displayed expression by

$$
C\left(\sum_{P \in T_{2}}\left|\left\langle f, \phi_{1 P}\right\rangle\right|^{2}\right)^{\frac{1}{2}} \leq C\left|I_{T}\right|^{\frac{1}{2}} \varepsilon .
$$

This completes the desired estimate for the $T_{2}$-part of (25) and thereby finishes the proof of (20).

\section{Eigenfunctions of one dimensional Schrödinger operators}

This chapter is devoted to an outline of an application of the theory of multilinear singular integrals with modulation symmetries to ordinary differential equations. Generally, multilinear operators are expected to appear as indvidual terms in Taylor series of non-linear operators, such as in the case of the Cauchy integral on Lipshitz curves mentioned in the discussion of uniform estimates in Chapter 1, or as higher order terms in iteration processes (which sometimes may coincide with a Taylor series). Here we shall be concerned with an example of an iteration process producing multilinear operators.

Consider the one dimensional time independent Schrödinger equation

$$
-f^{\prime \prime}(x)+V(x) f(x)=\lambda^{2} f(x) .
$$

If $V$ is constant equal to 0 , we have the constant coefficient ODE

$$
-f^{\prime \prime}(x)=\lambda^{2} f(x)
$$


whose general solution is a linear combination of the form

$$
a e^{i \lambda x}+b e^{-i \lambda x} \text {. }
$$

In particular, for real $\lambda$, all solutions of this ODE are bounded functions.

We are concerned with the question, under which smallness assumption on $V$ do we preserve the property of having - at least for almost all real $\lambda$ - only bounded solutions. This question is related to the spectral theory of the Schrödinger operator involved, in particular this boundedness property implies the existence of a large absolute continuous spectrum $[\mathbf{2 1}]$.

The conjecture is that $V \in L^{2}$ is sufficient:

Conjecture 3.1. If $V$ is real and $V \in L^{2}(\mathbb{R})$, then, for almost all real $\lambda$, all solutions of $(27)$ are in $L^{\infty}$.

The analogue statment for $V \in L^{r}(\mathbb{R})$ and $1 \leq r<2$ has been proved recently by Christ and Kiselev $[\mathbf{7}](r=1$ has been known for a long time, see [7] for a history of the subject, while $r$ close to 2 is the difficult region). The hypothetic corollary, that for $V$ as in the conjecture the interval $[0, \infty)$ is an essential support for the absolute continuous spectrum of the Schrödinger operator has been proved by different methods recently by Deift and Killip [11]; their methods however do not give any further information as to the truth of the above conjecture.

The approach of Christ and Kiselev, which we would like to reproduce here, is to expand the solutions of (27) into a formal series (called WKB) using an appropriate iteration scheme, and then prove good estimates on each term in the series.

We observe that there is an approximate solution to (27) of the form

$$
\phi(x)=e^{i \lambda x-\frac{i}{2 \lambda} \int_{0}^{x} V(y) d y}
$$

which can be obtained by inserting

$$
\phi(x)=e^{i \lambda x+\theta(x)}
$$

into the Schrödinger equation and neglecting the terms $\theta^{\prime \prime}$ and $\left(\theta^{\prime}\right)^{2}$ in the resulting equation for $\theta$. Observe that this is a slowly - varyingassumption on $\theta$.

Likewise, $\bar{\phi}$ is an approximate solution.

For an exact solution we set

$$
f(x)=a(x) \phi(x)+b(x) \overline{\phi(x)}
$$


with slowly varying $a$ and $b$. Here slowly varying means we impose a condition on $a$ and $b$ which makes the second order derivatives of $a$ and $b$ disappear (in the otherwise underdetermined equation for $a$ and $b$ ):

$$
f^{\prime}(x)=i \lambda a(x) \phi(x)-i \lambda b(x) \overline{\phi(x)} .
$$

The Schrödinger equation yields the following first order system of ODEs:

$$
\left(\begin{array}{l}
a \\
b
\end{array}\right)^{\prime}=\frac{i}{2 \lambda}\left(\begin{array}{cc}
0 & -V \bar{\phi}^{2} \\
V \phi^{2} & 0
\end{array}\right)\left(\begin{array}{l}
a \\
b
\end{array}\right) .
$$

We shall read this as an iteration scheme as follows:

$$
\left(\begin{array}{l}
a_{n+1}(x) \\
b_{n+1}(x)
\end{array}\right)=\left(\begin{array}{l}
1 \\
0
\end{array}\right)-\int_{x}^{\infty} \frac{i}{2 \lambda}\left(\begin{array}{cc}
0 & -V \bar{\phi}^{2} \\
V \phi^{2} & 0
\end{array}\right)\left(\begin{array}{l}
a_{n} \\
b_{n}
\end{array}\right) d y .
$$

Here we have imposed the boundary condition $(a(\infty), b(\infty))=(1,0)$. An appropriate different boundary condition gives a scheme for a linearly independent solution, but since the structure of the scheme is the same we shall not discuss other boundary conditions.

The above iteration scheme has the following formal solution

$$
\begin{aligned}
& a=\sum_{n=0}^{\infty}\left(\frac{1}{2 \lambda}\right)^{2 n} T_{2 n}(V, \ldots, V) \\
& b=-i \sum_{n=1}^{\infty}\left(\frac{1}{2 \lambda}\right)^{2 n-1} T_{2 n-1}(V, \ldots, V)
\end{aligned}
$$

where $T_{0} \equiv 1$ and

$$
T_{n}\left(f_{1}, \ldots, f_{n}\right)(x)=\int_{x}^{\infty} \int_{t_{1}}^{\infty} \cdots \int_{t_{n-1}}^{\infty} \prod_{j=1}^{n} \phi^{2(-1)^{n-j}}\left(t_{j}\right) f_{j}\left(t_{j}\right) d t_{j} .
$$

These are multilinear operators, and ultimately we are interested in controlling $T\left(f_{1}, \ldots, f_{n}\right)$ in the sup norm, given $L^{2}$ control over the functions $f_{i}$.

Recall that the above lines have an implicit $\lambda$ dependence. Homogeneity considerations suggest the following conjectured bound

$$
\left\|\sup _{x}\left|T_{n}\left(f_{1}, \ldots, f_{n}\right)(x, \lambda)\right|\right\|_{L^{\frac{2}{n}(\lambda)}} \leq C_{n}\left\|f_{1}\right\|_{2} \ldots\left\|f_{n}\right\|_{2} .
$$

While such a bound does not yet guarantee summability of the iteration series, we shall solely concentrate on this bound for the rest of this discussion. Summability requires additional arguments, which use that in this specific application all the functions $f_{i}$ are the same, see [7]. 
To simplify the discussion we shall replace the WKB phase and its various powers by a simple exponential phase $e^{2 \pi i \lambda\left(t_{1}+\cdots+t_{n}\right)}$. The WKB phase is an approximation to an exponential and neglecting it is a minor modification. Neglecting the minus signs in the exponent - as pointed out by M. Christ - is a more subtle change, it passes form a degenerate to a nondegenerate stiuation in the sense explained in the first chapter of this survey article. However, it seems reasonable to study the nondegenerate situation first. Also, to comply with our setup in Chapter 1, we shall replace $f_{i}$ by $\widehat{f}_{i}$. We can neglect the Fourier transform on the right hand side of (28) since it is an isometry in $L^{2}$.

Thus we are looking at the multi(sub)linear maximal operator

(29) $T_{\max }\left(f_{1}, \ldots, f_{n}\right)(x)$

$$
=\sup _{\xi}\left|\int_{\xi<\xi_{1}<\xi_{2}<\cdots<\xi_{n}} e^{2 \pi i x\left(\xi_{1}+\cdots+\xi_{n}\right)} \widehat{f}_{1}\left(\xi_{1}\right) \ldots \widehat{f}_{n}\left(\xi_{n}\right) d \xi_{1} \ldots d \xi_{n}\right|
$$

and, taking $\xi=-\infty$ instead of a supremum over $\xi$, a linear operator

$$
\begin{aligned}
& T\left(f_{1}, \ldots, f_{n}\right)(x) \\
& \quad=\int_{\xi_{1}<\xi_{2}<\cdots<\xi_{n}} e^{2 \pi i x\left(\xi_{1}+\cdots+\xi_{n}\right)} \widehat{f}_{1}\left(\xi_{1}\right) \ldots \widehat{f}_{n}\left(\xi_{n}\right) d \xi_{1} \ldots d \xi_{n} .
\end{aligned}
$$

This linear operator is a multiplier operator of the type (11) discussed in Chapter 1, the multiplier being the characteristic function of an infinite simplex $\xi_{1}<\cdots<\xi_{n}$.

We shall discuss these operators for small values of $n$.

If $n=1$, the linear operator is just the identity operator

$$
T_{1} f(x)=\int_{-\infty}^{\infty} \widehat{f}(\xi) e^{2 \pi i \lambda \xi} d \xi=f(x)
$$

while the associated maximal operator is essentially the Carleson operator

$$
T_{\max } f(x)=\sup _{x}\left|\int_{x}^{\infty} \widehat{f}(\xi) e^{2 \pi i \lambda \xi} d \xi\right| .
$$

Both operators are bounded in $L^{2}$, which is the desired estimate. Already in this simplest case the modulation invariant theory enters the picture in the form of Carleson's theorem. We remark that doing an analogue discussion of the work of Christ and Kiselev, the estimates needed here are a bound for Carleson's operator given that $\widehat{f}$ is in $L^{p}$ ( $f$ is in a Wiener type space) with $p<2$, which is a classical bound known as long ago as [41], long before the proof of Carleson's theorem. 
If $n=2$, then the linear operator is essentially the bilinear Hilbert transform

$$
T\left(f_{1}, f_{2}\right)(x)=\int_{-\infty}^{\infty} \int_{\xi_{1}}^{\infty} \prod_{j=1}^{2} \widehat{f}\left(\xi_{j}\right) e^{2 \pi i \lambda \xi_{j}} d \xi_{j},
$$

while the maximal operator is:

$$
T_{\max }\left(f_{1}, f_{2}\right)(x)=\sup _{x}\left|\int_{x}^{\infty} \int_{\xi_{1}}^{\infty} \prod_{j=1}^{2} \widehat{f}\left(\xi_{j}\right) e^{2 \pi i \lambda \xi_{j}} d \xi_{j}\right| .
$$

The bilinear Hilbert transform satisfies the desired bound $L^{2} \times L^{2} \rightarrow L^{1}$, for the maximal operator this is as of yet unknown (work in progress). Since the Carleson operator and the bilinear Hilbert transform have roughly the same complexity, we may expect that the maximal operator for $n=2$ has the same complexity as the linear operator for $n=3$ :

$$
T\left(f_{1}, f_{2}, f_{3}\right)(x)=\int_{-\infty}^{\infty} \int_{\xi_{1}}^{\infty} \int_{\xi_{2}}^{\infty} \prod_{j=1}^{3} \widehat{f}\left(\xi_{j}\right) e^{2 \pi i \lambda \xi_{j}} d \xi_{j} .
$$

We shall call this trilinear operator the "biest". (This is german spelling for "beast", and it allows for generalizations "triest" and "multiest".)

The biest is a multiplier operator whose multiplier is given by the characteristic function of the convex hull of

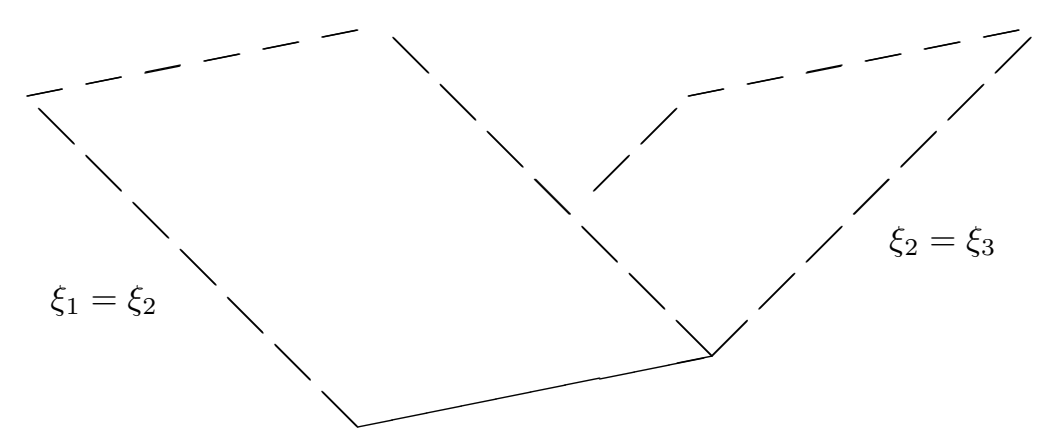


Each of the planes $\xi_{1}=\xi_{2}$ and $\xi_{2}=\xi_{3}$ by themselves correspond to operators

$$
B\left(f_{1}, f_{2}\right) \cdot f_{3}, \quad f_{1} \cdot B\left(f_{2}, f_{3}\right),
$$

where $B$ is a variant of the bilinear Hilbert transform. Both planes are degenerate in the sense of Theorem 1.3. Also, in the notation of that theorem, we have $k=2$ and $n=4$, so the condition $2 k<n$ is violated. However, due to the above splitting, we certainly obtain a whole range of $L^{p}$ estimates for both operators.

However, no such splitting is available for the biest itself. So no $L^{p}$ estimates for the biest are immediately evident. However, we have the following theorem [34], [35], which is the first progress (beyond what was discussed in Chapter 1) towards understanding of the $L^{2}$ theory of the eigenfunction expansions of Schrödinger operators:

Theorem 3.2. Define $\Lambda\left(f_{1}, f_{2}, f_{3}, f_{4}\right)$ to be

$$
\int_{\xi_{1}+\xi_{2}+\xi_{3}+\xi_{4}=0, \xi_{1}<\xi_{2}, \xi_{2}<\xi_{3}} \prod_{j=1}^{4} \widehat{f}_{j}\left(\xi_{j}\right) d \mu .
$$

Then we have the a priori estimates

$$
\left|\Lambda\left(f_{1}, f_{2}, f_{3}, f_{4}\right)\right| \leq C_{p_{1}, p_{2}, p_{3}, p_{4}} \prod_{j=1}^{4}\left\|f_{j}\right\|_{p_{j}}
$$

as long as

$$
\sum_{j=1}^{4} \frac{1}{p_{j}}=1, \quad 1<p_{j}<\infty .
$$

Moreover, we have the following types $\left(p_{1}, p_{2}, p_{3}, p_{4}\right)$ provided at most one of the $p_{i}$ is negative,

$$
\left(\frac{1}{p_{1}}, \frac{1}{p_{2}}, \frac{1}{p_{3}}, \frac{1}{p_{4}}\right) \in D^{\prime} \cap D,
$$

where $D^{\prime}$ is the open convex hull of the points

$$
\left(\frac{1}{2}, 1,1,-\frac{3}{2}\right),\left(-\frac{1}{2}, 1, \frac{1}{2}, 0\right),\left(-\frac{1}{2}, \frac{1}{2}, 1,0\right)
$$

and their images under the index permutations $1 \rightarrow 2$ and $3 \rightarrow 4$ (12 points altogether) and $D$ is the image of $D^{\prime}$ under the index permutations $1 \rightarrow 3$.

In particular, $\Lambda$ is of type $(2,2,2,-2)$. 
We remark that the multiplier of the biest can be cut by a smooth cutoff function into two pieces symmetric under the index permutations $\xi_{1} \rightarrow \xi_{2}$, one of them has the singularities $\xi_{1}=\xi_{2}$ and $\xi_{1}=$ $\xi_{2}=\xi_{3}=a_{4} \xi_{4}$. These two regions correspond to the regions $D^{\prime}$ and $D$ of estimates in the theorem.

We remark that this theorem has analogues for more general multipliers in the spirit of Gilbert and Nahmod [13], however, a tensor product constraint as discussed in [15] appears.

The series of multilinear operators and maximal operators discussed above is currently under active research, and there may be hope that the Conjecture 3.1 can be solved along these lines.

Addendum. During the process of this article being published, it was discovered in joint work with Muscalu and Tao that the multilinear operators which actually appear in the series expansion of eigenfunctions of Schrödinger operators are not bounded in $L^{2}$. This result will appear elsewhere.

\section{References}

[1] J. Barrionuevo And M. T. LACEY, A weak type orthogonality principle, Preprint (1997).

[2] J. Bourgain, Pointwise ergodic theorems for arithmetic sets, Inst. Hautes Études Sci. Publ. Math. 69 (1989), 5-45.

[3] A. P. CALDERón, Commutators of singular integral operators, Proc. Nat. Acad. Sci. U.S.A. 53 (1965), 1092-1099.

[4] A. P. Calderón, Cauchy integrals on Lipschitz curves and related operators, Proc. Nat. Acad. Sci. U.S.A. 74(4) (1977), 1324-1327.

[5] A. P. Calderón And A. Zygmund, A note on the interpolation of linear operations, Studia Math. 12 (1951), 194-204.

[6] L. CARleson, On convergence and growth of partial sums of Fourier series, Acta Math. 116 (1966), 135-157.

[7] M. Christ And A. Kiselev, WKB asymptotic behavior of almost all generalized eigenfunctions for one-dimensional Schrödinger operators with slowly decaying potentials, J. Funct. Anal. 179(2) (2001), 426-447.

[8] M. Christ And A. Kiselev, WKB and spectral analysis of onedimensional Schrödinger operators with slowly varying potentials, Comm. Math. Phys. 218(2) (2001), 245-262.

[9] R. R. Coifman, A. McIntosh and Y. Meyer, L'intégrale de Cauchy définit un opérateur borné sur $L^{2}$ pour les courbes lipschitziennes, Ann. of Math. (2) 116(2) (1982), 361-387. 
[10] R. R. CoIfman And Y. Meyer, Fourier analysis of multilinear convolutions, Calderón's theorem, and analysis of Lipschitz curves, in: "Euclidean harmonic analysis" (Proc. Sem., Univ. Maryland, College Park, Md., 1979), Lecture Notes in Math. 779, Springer, Berlin, 1980, pp. 104-122.

[11] P. Deift AND R. KILliP, On the absolutely continuous spectrum of one-dimensional Schrödinger operators with square summable potentials, Comm. Math. Phys. 203(2) (1999), 341-347.

[12] C. Fefferman, Pointwise convergence of Fourier series, Ann. of Math. (2) 98 (1973), 551-571.

[13] J. E. Gilbert and A. R. Nahmod, Boundedness of bilinear operators with nonsmooth symbols, Math. Res. Lett. 7(5-6) (2000), 767-778.

[14] J. E. Gilbert And A. R. NAhmod, Hardy spaces and a Walsh model for bilinear cone operators, Trans. Amer. Math. Soc. 351(8) (1999), 3267-3300.

[15] L. Grafakos And N. J. Kalton, The Marcinkiewicz multiplier condition for bilinear operators, Studia Math. 146(2) (2001), $115-156$.

[16] L. Grafakos AND X. Li, Uniform bounds for the bilinear Hilbert transform. I, Preprint (2000).

[17] L. Grafakos And X. Li, The disc as multiplier, Preprint (2000).

[18] R. A. Hunt, On the convergence of Fourier series, in: "Orthogonal Expansions and their Continuous Analogues" (Proc. Conf., Edwardsville, Ill., 1967), Southern Illinois Univ. Press, Carbondale, Ill, 1968, pp. 235-255.

[19] S. JAnson, On interpolation of multilinear operators, in: "Function spaces and applications" (Lund, 1986), Lecture Notes in Math. 1302, Springer, Berlin, 1988, pp. 290-302.

[20] C. E. Kenig And E. M. Stein, Multilinear estimates and fractional integration, Math. Res. Lett. 6(1) (1999), 1-15.

[21] A. Kiselev, Y. Last And B. Simon, Modified Prüfer and EFGP transforms and the spectral analysis of one-dimensional Schrödinger operators, Comm. Math. Phys. 194(1) (1998), 1-4.

[22] A. Kolmogorov, Sur les fonctions harmoniques conjuguées e les sèries de Fourier, Fund. Math. 7 (1925), 23-28.

[23] M. T. LACEY, The bilinear Hilbert transform is pointwise finite, Rev. Mat. Iberoamericana 13(2) (1997), 411-469. 
[24] M. T. LACEY, The bilinear maximal functions map into $L^{p}$ for $2 / 3<p \leq 1$, Ann. of Math. (2) 151(1) (2000), 35-57.

[25] M. T. LACEY AND C. M. Thiele, $L^{p}$ estimates on the bilinear Hilbert transform for $2<p<\infty$, Ann. of Math. (2) 146(3) (1997), 693-724.

[26] M. T. Lacey and C. M. Thiele, $L^{p}$ estimates for the bilinear Hilbert transform, Proc. Nat. Acad. Sci. U.S.A. 94(1) (1997), $33-35$.

[27] M. T. Lacey and C. M. Thiele, On Calderón's conjecture, Ann. of Math. (2) 149(2) (1999), 475-496.

[28] M. T. Lacey and C. M. Thiele, On Calderón's conjecture for the bilinear Hilbert transform, Proc. Natl. Acad. Sci. USA 95(9) (1998), 4828-4830.

[29] M. T. LaCey and C. M. Thiele, A proof of boundedness of the Carleson operator, Math. Res. Lett. 7(4) (2000), 361-370.

[30] X. LI, Uniform bounds for the bilinear Hilbert transform. II, Preprint (2000).

[31] Y. Meyer and R. R. Coifman, "Ondelettes et opérateurs. III", Opérateurs multilinéaires, Actualités Mathématiques, Hermann, Paris, 1991.

[32] C. Muscalu, $L^{p}$ estimates for multipliers given by singular symbols, PhD Thesis, Brown University (2000).

[33] C. Muscalu, T. Tao and C. M. Thiele, Multilinear operators given by singular multipliers, J. Amer. Math. Soc. 15(2) (2002), 469-496.

[34] C. Muscalu, T. Tao and C. M. Thiele, $L^{p}$ estimates for the biest I. The Walsh case, Preprint (2001).

[35] C. Muscalu, T. Tao and C. M. Thiele, $L^{p}$ estimates for the biest II. The Fourier case, Preprint (2001).

[36] M. Riesz, Sur les fonctions conjuguées, Math. Z. 27 (1927), $218-244$.

[37] E. M. STEIN, "Harmonic analysis: real-variable methods, orthogonality, and oscillatory integrals", Princeton Mathematical Series 43, Monographs in Harmonic Analysis III, Princeton University Press, Princeton, NJ, 1993.

[38] C. M. Thiele, Time-frequency analysis in the discrete phase plane, $\mathrm{PhD}$. Thesis, Yale University (1995).

[39] C. M. ThiELE, The quartile operator and pointwise convergence of Walsh series, Trans. Amer. Math. Soc. 352(12) (2000), 5745-5766.

[40] C. M. Thiele, "On the Bilinear Hilbert transform", Universität Kiel, Habilitationsschrift, 1998. 
[41] A. Zygmund, A remark on Fourier transforms, Math. Proc. Cambridge Philos. Soc. 32 (1936), 321-327.

Department of Mathematics

University of California

Los Angeles, CA 90024

U.S.A.

E-mail address: thiele@math.ucla.edu 